\title{
SPECTRAL LINE DE-CONFUSION IN AN INTENSITY MAPPING SURVEY
}

\author{
Yun-Ting Cheng $^{1,2}$, Tzu-Ching Chang ${ }^{2}, \mathrm{James}$ Bock $^{1,3}$, C. Matt Bradford ${ }^{1,3}$, and Asantha Cooray ${ }^{4}$ \\ ${ }^{1}$ California Institute of Technology, 1200 E. California Boulevard, Pasadena, CA 91125, USA; ycheng3@ caltech.edu \\ ${ }^{2}$ Institute of Astronomy and Astrophysics, Academia Sinica, 1 Roosevelt Road, Section 4, Taipei, 10617, Taiwan \\ ${ }^{3}$ NASA Jet Propulsion Laboratory, 4800 Oak Grove Drive, Pasadena, CA 91109, USA \\ ${ }^{4}$ Department of Physics and Astronomy, University of California, Irvine, CA 92697, USA \\ Received 2016 April 26; revised 2016 September 19; accepted 2016 September 26; published 2016 November 30
}

\begin{abstract}
Spectral line intensity mapping (LIM) has been proposed as a promising tool to efficiently probe the cosmic reionization and the large-scale structure. Without detecting individual sources, LIM makes use of all available photons and measures the integrated light in the source confusion limit to efficiently map the three-dimensional matter distribution on large scales as traced by a given emission line. One particular challenge is the separation of desired signals from astrophysical continuum foregrounds and line interlopers. Here we present a technique to extract large-scale structure information traced by emission lines from different redshifts, embedded in a threedimensional intensity mapping data cube. The line redshifts are distinguished by the anisotropic shape of the power spectra when projected onto a common coordinate frame. We consider the case where high-redshift [C II] lines are confused with multiple low-redshift CO rotational lines. We present a semi-analytic model for [C II] and CO line estimates based on the cosmic infrared background measurements, and show that with a modest instrumental noise level and survey geometry, the large-scale [C II] and CO power spectrum amplitudes can be successfully extracted from a confusion-limited data set, without external information. We discuss the implications and limits of this technique for possible LIM experiments.
\end{abstract}

Key words: cosmology: observations - cosmology: theory - dark ages, reionization, first stars - large-scale structure of universe

\section{INTRODUCTION}

Line intensity mapping (LIM) has emerged as a promising tool to probe the three-dimensional structure of the universe. Several emission lines have been proposed to uniquely trace the cosmic reionization process, revealing properties of the ionizing sources and the intergalactic medium at high redshifts, and to efficiently map the large-scale matter distribution in a large cosmic volume, suitable for cosmological studies at lower redshifts.

In contrast to traditional large-scale structure surveys, intensity mapping (IM) operates in the confusion-limited regime without thresholding to identify individual sources; rather, IM makes use of integrated light emission from all sources, including unresolved faint galaxies, to statistically measure properties of the light tracers and the underlying matter distribution. In addition, with LIM, where the tracer is a particular spectral line emission, the three-dimensional matter distribution can be faithfully represented on large cosmic scales. The $21 \mathrm{~cm}$ hyperfine emission from neutral hydrogen (Scott \& Rees 1990; Madau et al. 1997; Chang et al. 2008; Wyithe \& Loeb 2008), the CO rotational lines (Righi et al. 2008; Visbal \& Loeb 2010; Carilli 2011; Gong et al. 2011; Lidz et al. 2011; Pullen et al. 2013; Breysse et al. 2014; Mashian et al. 2015; Keating et al. 2015; Li et al. 2016), the [C II] $157.7 \mu \mathrm{m}$ fine structure line (Gong et al. 2012; Uzgil et al. 2014; Silva et al. 2015; Yue et al. 2015), and the Ly $\alpha$ emission line (Silva et al. 2013; Gong et al. 2014; Pullen et al. 2014; Comaschi \& Ferrara 2016) are among the most studied such tracers in the LIM regime.

One of the main challenges in an LIM experiment is the separation of signals from the astrophysical foreground continuum emissions and line interlopers. The continuum foreground issue has been studied mostly in the context of $21 \mathrm{~cm} \mathrm{IM}$, where the Galactic and extragalactic synchrotron and free-free radiations overwhelm the expected signals by several orders of magnitude (e.g., Furlanetto et al. 2006; Morales et al. 2006; Bowman et al. 2009; Chapman et al. 2012; Liu \& Tegmark 2012; Parsons et al. 2012; Switzer et al. 2015); line interlopers, on the other hand, are a pressing issue for other line probes in the electromagnetic spectrum that is crowded with other line features.

Several studies have proposed strategies for deblending lines in an IM survey, by means of masking and cross-correlation. The masking technique makes use of an external galaxy catalog from galaxy surveys to identify and remove bright sources in order to reduce potential foreground contaminations (Breysse et al. 2015; Silva et al. 2015; Yue et al. 2015). On the other hand, cross-correlation of an LIM survey with an external data set tracing the same cosmic volume can help extract signals of interest; the method has been proposed, in particular, in the studies of reionization (Lidz et al. 2009; Visbal \& Loeb 2010; Gong et al. 2012, 2014; Chang et al. 2015; Silva et al. 2015), and has been successfully applied to extract LIM signals at lower redshifts against continuum foregrounds (Chang et al. 2010; Masui et al. 2013; Croft et al. 2016). Aside from these two methods, Kogut et al. (2015) makes use of the companion lines to directly identify [C II] line intensity in each voxel. de Putter et al. (2014) propose to use angular fluctuations of the light to reconstruct the 3D source luminosity density.

In an IM experiment, the intrinsic observing coordinates are angular and spectral coordinates defined by the instrument and survey geometry, which, given a known redshift, are mapped into comoving coordinates in the redshift space, before a power spectrum is computed. In the linear regime, any line tracers 
supposedly follow the matter distribution, which is isotropic in their respective real-space coordinates. If, however, without a priori knowledge of redshifts, lines at different redshifts embedded in an observing volume are blindly projected into the same comoving coordinates, they will exhibit different anisotropic 3D shapes in that frame, due to the incorrect redshift projection. This is key to the line separation technique we employ in this paper. The idea has been previously suggested by Visbal \& Loeb (2010) and Gong et al. (2014), and recently investigated by Lidz \& Taylor (2016).

To demonstrate this technique, we consider a 3D LIM observing volume with high-redshift [C II] emissions blended with multiple lower-redshift $\mathrm{CO}$ rotational lines. We present a halo-model-based formalism to estimate [C II] and $\mathrm{CO}$ line strengths and power spectra across redshifts. After projecting the observed volume onto a common comoving frame, the resulting total power spectrum is a superposition of [C II] and $\mathrm{CO}$ power spectra at different redshifts, each with a different but predictable 3D shape due to the projection which we use as templates. We generate simulated data and use the Markov Chain Monte Carlo (MCMC) formalism to extract power spectrum parameters based on the templates.

The paper is organized as follows. In Section 2, we describe a model to estimate the [C II] and $\mathrm{CO}$ power spectra across redshifts, and the formalism for expressing the 3D power spectra of both lines in the comoving frame of [C II]. In Section 3, we discuss the details of the template fitting and MCMC implementation. The results are presented in Section 4. In Section 5, we discuss the implication and limitation of our method, and conclude in Section 6. Throughout this paper, we consider a flat $\Lambda$ CDM cosmology with $n_{s}=0.97, \sigma_{8}=0.82$, $\Omega_{m}=0.26, \Omega_{b}=0.049, \Omega_{\Lambda}=0.69$, and $h=0.68$, consistent with the latest measurement from Planck (Planck Collaboration et al. 2015).

\section{POWER SPECTRUM MODELING}

Here we provide an estimate of the [C II] and $\mathrm{CO}$ power spectra as a function of redshift. Our modeling is based on the halo-model formalism (Cooray \& Sheth 2002). With the [C II] and $\mathrm{CO}$ luminosity $\left(L_{\mathrm{C} \text { II }}, L_{\mathrm{CO}}\right)$ and halo mass $(M)$ relations, the power spectrum of a spectral line intensity field can be calculated with the halo model.

We build our model (referred to as the CIB model hereafter) based on Planck Collaboration et al. (2014); the authors fitted the $L_{\mathrm{IR}}$ and and halo mass relation with cosmic infrared background (CIB) emission as measured by Planck. Details of the CIB model are provided in Appendix A.1.

Then we connect the $L_{\mathrm{IR}}$ to $L_{\mathrm{C} \text { II }}$ and $L_{\mathrm{CO}}$. We adopt these relations based on observations and simulations in the literature. See Appendix A.2 for more details.

\subsection{Power Spectrum with Halo Model}

With the luminosity-halo mass relation at hand, we derive the power spectrum in the halo-model framework. The comoving power spectrum consists of the one-halo and twohalo terms, which account for the correlation within the halos and between halos, respectively. The one-halo term of the [C II]

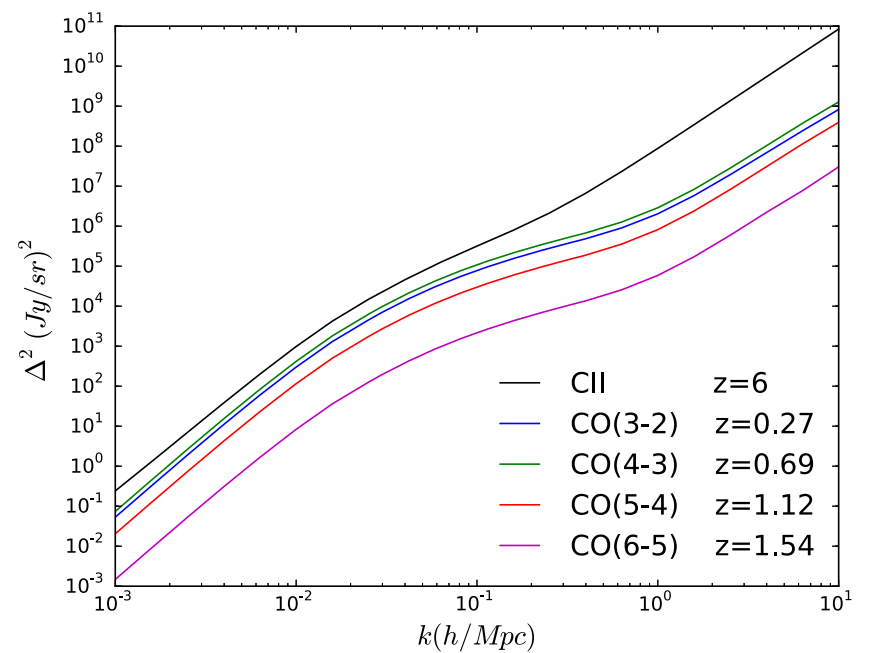

Figure 1. Comoving isotropic power spectra of [C II] at $z=6$ and $\mathrm{CO}$ interlopers at lower redshfits in the same observing frequency range.

or $\mathrm{CO}$ line is given by

$$
\begin{aligned}
P_{1 h}^{\text {line }}(k, z)= & \int_{M_{\min }}^{M_{\max }} d M \frac{d N}{d M}|u(k \mid M)|^{2} \\
& \times\left(\frac{L_{\text {line }}(M, z)}{4 \pi D_{L}^{2}(z)} y_{\text {line }}(z) D_{A}^{2}(z)\right)^{2},
\end{aligned}
$$

where $L_{\text {line }}(M, z)$ is the luminosity of [C II] or CO for a given halo mass $M$ at redshift $z, D_{L}$ is the proper luminosity distance, $D_{A}$ is the comoving angular diameter distance, $u(k \mid M)$ is the Fourier transform of the normalized halo density profile, and we adopt the NFW profile in this work (Navarro et al. 1996). $y_{\text {line }}(z) \equiv d \chi / d \nu=\lambda_{\text {line }}(1+z)^{2} / H(z), \quad$ where $\quad \chi \quad$ is the comoving distance, $H$ is the Hubble parameter, $\nu$ is the observed frequency, and $\lambda_{\text {line }}$ is the rest-frame wavelength of the line.

The two-halo term can be written as

$$
\begin{aligned}
P_{2 h}^{\mathrm{line}}(k, z)= & {\left[\int_{M_{\min }}^{M_{\max }} d M \frac{d N}{d M}|u(k \mid M)| b(M, z)\right.} \\
& \left.\times\left(\frac{L_{\mathrm{line}}(M, z)}{4 \pi D_{L}^{2}(z)} y_{\text {line }}(z) D_{A}^{2}(z)\right)\right]^{2} P_{\operatorname{lin}}(k),
\end{aligned}
$$

where $b(M, z)$ is the halo bias (Sheth \& Tormen 1999), and $P_{\operatorname{lin}}(k)$ is the linear matter power spectrum.

We also consider the shot noise power spectrum due to the discretization of sources:

$$
P_{\mathrm{sh}}^{\mathrm{line}}(z)=\int_{M_{\min }}^{M_{\max }} d M \frac{d N}{d M}\left(\frac{L_{\mathrm{line}}(M, z)}{4 \pi D_{L}^{2}(z)} y_{\text {line }}(z) D_{A}^{2}(z)\right)^{2} .
$$

The total comoving power spectrum is thus

$$
P_{\mathrm{tot}}^{\text {line }}(k, z)=P_{1 h}^{\mathrm{line}}(k, z)+P_{2 h}^{\text {line }}(k, z)+P_{\mathrm{sh}}^{\text {line }}(z) .
$$

Figure 1 shows the comoving isotropic power spectrum of [C II] at $z=6$ based on the CIB model, and the lower-redshift CO power spectra, which overlap in the same observing frequency range. 


\subsection{D Power Spectrum}

The [C II] and CO power spectra $P(k)$ is isotropic in realspace on large scales, so the power spectra only depend on $|\boldsymbol{k}|$ but not the direction of the $\boldsymbol{k}$ vector. However, an anisotropic feature comes in due to observational effects, which introduce the dependence on $\mu$, the cosine of the angle between the $\boldsymbol{k}$ vector, and the line-of-sight direction. Below we discuss the projection effect and the redshift space distortions (RSDs) that give rise to the anisotropy in the $3 \mathrm{D}$ power spectrum.

\subsubsection{Projection Effect}

In a redshifted [C II] IM experiment, a CO line signal from redshift $z_{\mathrm{CO}}$ will blend with the [C II] signal from $z_{\mathrm{C} \text { II }}$ if they have the same observing frequency. The redshifts of these two lines follow

$$
\nu_{\mathrm{obs}}=\frac{\nu_{\mathrm{C}}}{\left(1+z_{\mathrm{CII}}\right)}=\frac{\nu_{\mathrm{CO}}}{\left(1+z_{\mathrm{CO}}\right)},
$$

where $\nu_{\mathrm{obs}}$ is the observed frequency, and $\nu_{\mathrm{C}}$ II and $\nu_{\mathrm{CO}}$ are the rest-frame frequencies of the $[\mathrm{C} \mathrm{II}]$ and $\mathrm{CO}$ lines, which are $1902 \mathrm{GHz}$ and $115 \mathrm{JGHz}$ for the $J$ to $J-1$ transition, respectively.

Both [C II] and $\mathrm{CO}$ power spectra are isotropic in their respective comoving frames. However, in the confusion limit, we may incorrectly project the observed $[\mathrm{C} \mathrm{II}]$ and $\mathrm{CO}$ signals onto the comoving frame of $[\mathrm{C} \mathrm{II}]$ for the power spectrum calculation. In this case, the $\mathrm{CO}$ power spectrum is no longer isotropic. This is caused by the different redshift projection factors parallel and perpendicular to the line of sight, which makes the $\mathrm{CO} 3 \mathrm{D}$ data cube stretched more in one direction than the other. Below we provide the formalism for calculating the $3 \mathrm{D}$ power spectrum of a low $-z \mathrm{CO}$ signal projected onto the high- $z$ [C II] comoving frame.

The projection in the direction parallel to the line of sight can be derived by considering an observed frequency range $d \nu_{\text {obs }}$, which corresponds to either the [C II] from a comoving size $R_{\mathrm{C} \text { II }}^{\|}$at $z_{\mathrm{C} \text { II }}$, or the $\mathrm{CO}$ signal with comoving size $R_{\mathrm{CO}}^{\|}$at $z_{\mathrm{CO}}$. The relation between $R_{\mathrm{C} \text { II }}^{\|}$and $R_{\mathrm{CO}}^{\|}$is

$$
\begin{aligned}
\frac{R_{\mathrm{CO}}^{\|}}{R_{\mathrm{C} \text { II }}^{\|}} & =\frac{d \chi\left(z_{\mathrm{CO}}\right) / d \nu_{\mathrm{obs}}}{d \chi\left(z_{\mathrm{C} \text { II }}\right) / d \nu_{\mathrm{obs}}}=\frac{y_{\mathrm{CO}}\left(z_{\mathrm{CO}}\right)}{y_{\mathrm{C} \text { II }}\left(z_{\mathrm{C} \mathrm{II}}\right)} \\
& =\frac{\lambda_{\mathrm{CO}}\left(1+z_{\mathrm{CO}}\right)^{2} / H\left(z_{\mathrm{CO}}\right)}{\lambda_{\mathrm{C} \text { II }}\left(1+z_{\mathrm{C} \text { II }}\right)^{2} / H\left(z_{\mathrm{C} \mathrm{II}}\right)} \\
& =\frac{\left(1+z_{\mathrm{CO}}\right) / H\left(z_{\mathrm{CO}}\right)}{\left(1+z_{\mathrm{C} \mathrm{II}}\right) / H\left(z_{\mathrm{C} \text { II }}\right)} .
\end{aligned}
$$

Since Fourier wavenumber $k^{\|} \propto 1 / R^{\|}$, we obtain

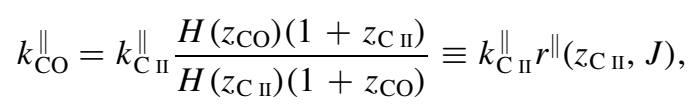

where $J$ labels the CO transition from $J$ to $J-1$, and $r^{\|}\left(z_{\mathrm{C}}, J\right)$ is the conversion factor for projecting the scale at $z_{\mathrm{CO}}$ to $z_{\mathrm{C} \text { II }}$ in the parallel direction.

The transverse scale of $\mathrm{CO}$ will be projected to the scale of [C II] corresponding to the same observed angle $\theta$. Hence, the scale conversion relation in the perpendicular direction is

$$
\theta=\frac{R_{\mathrm{CO}}^{\perp}}{D_{A}\left(z_{\mathrm{CO}}\right)}=\frac{R_{\mathrm{C} \text { II }}^{\perp}}{D_{A}\left(z_{\mathrm{CII}}\right)}
$$

where $D_{A}$ is the comoving angular diameter distance. Thus

$$
k_{\mathrm{CO}}^{\perp}=k_{\mathrm{C} \text { II }}^{\perp} \frac{D_{A}\left(z_{\mathrm{C} \text { II }}\right)}{D_{A}\left(z_{\mathrm{CO}}\right)} \equiv k_{\mathrm{C} \text { II }}^{\perp} r^{\perp}\left(z_{\mathrm{C} \mathrm{II}}, J\right),
$$

where $r^{\perp}\left(z_{\mathrm{CII}}, J\right)$ is the conversion factor for projecting the scale at $z_{\mathrm{CO}}$ to $z_{\mathrm{C} \text { II }}$ in the perpendicular direction. Besides the shift in $k$ value, the projection changes the comoving voxel volume $V_{\text {vox }}$ and induces an amplitude change in the power spectrum. Since the power spectrum is proportional to $1 / V_{\text {vox }}$ at fixed intensity fluctuation, the projected $\mathrm{CO}$ power spectrum needs to be multiplied by the change in voxel volume $r^{\|}\left(r^{\perp}\right)^{2}$.

The $\mathrm{CO}$ projected power spectrum $P_{\mathrm{CO}}^{\mathrm{prj}}$ can thus be written as

$$
P_{\mathrm{CO}}^{\mathrm{prj}}\left(k_{\mathrm{C}}^{\perp}, k_{\mathrm{C} \mathrm{II}}^{\|}, z_{\mathrm{C} \text { II }}, J\right)=r^{\|}\left(r^{\perp}\right)^{2} P_{\mathrm{CO}}\left(k_{\mathrm{CO}}, z_{\mathrm{CO}}\right),
$$

where $k_{\mathrm{CO}}=\sqrt{\left(k_{\mathrm{CO}}^{\perp}\right)^{2}+\left(k_{\mathrm{CO}}^{\|}\right)^{2}}$, and $P_{\mathrm{CO}}$ is the comoving CO power spectrum.

For completeness, we also write down the [C II] power spectrum in the same coordinate,

$$
P_{\mathrm{C} \text { II }}^{\mathrm{prj}}\left(k_{\mathrm{C} \text { II }}^{\perp}, k_{\mathrm{C} \text { II }}^{\|}, z_{\mathrm{C} \text { II }}\right)=P_{\mathrm{C}_{\text {II }}}\left(k_{\mathrm{C}_{\text {II }}}, z_{\mathrm{C}_{\text {II }}}\right) \text {, }
$$

where $k_{\mathrm{C} \text { II }}=\sqrt{\left(k_{\mathrm{C} \text { II }}^{\perp}\right)^{2}+\left(k_{\mathrm{CII}}^{\|}\right)^{2}}$.

\subsubsection{Redshift Space Distortions}

Here we incorporate the RSD effects. We consider the linear Kaiser effect (Kaiser 1987) describing the coherent motion of structure growth on large scales, which enhances the power spectrum, and the suppression on small scales due to the nonlinear virial motion, which we write as an exponential damping term (Peacock 1992). The comoving one-halo and two-halo power spectrum can be written as (White 2001)

$$
\begin{aligned}
& P_{1 h}^{\operatorname{line}}(k, \mu, z)\left(1+\beta \mu^{2}\right)^{2} \times \int_{M_{\min }}^{M_{\max }} d M \frac{d N}{d M} \\
&= {\left[\left(\frac{L_{\text {line }}(M, z)}{4 \pi D_{L}^{2}(z)} y_{\text {line }}(z) D_{A}^{2}(z)\right)^{2}\right.} \\
& \times\left.|u(k \mid M)|^{2} e^{-\left(k \sigma_{v} \mu\right)^{2} / 2}\right], \\
& P_{2 h}^{\text {line }}(k, \mu, z) \\
&=P_{\operatorname{lin}}(k)\left(1+\beta \mu^{2}\right)^{2} \times\left\{\int_{M_{\min }}^{M_{\max }} d M \frac{d N}{d M}\right. \\
& \times\left[\left(\frac{L_{\text {line }}(M, z)}{4 \pi D_{L}^{2}(z)} y_{\text {line }}(z) D_{A}^{2}(z)\right)\right. \\
&\left.\left.\times|u(k \mid M)| b(M, z) e^{-\left(k \sigma_{v} \mu\right)^{2} / 2}\right]\right\}^{2},
\end{aligned}
$$

where $\left(1+\beta \mu^{2}\right)^{2}$ is the Kaiser effect and $e^{-\left(k \sigma_{v} \mu\right)^{2} / 2}$ is the exponential damping term. $\beta \equiv f / \bar{b}_{\text {line }}$, where $\mathrm{f}=\mathrm{d} \ln \mathrm{D} / \mathrm{d} \ln$ a is the logarithm derivative of the linear growth rate $D(z)$ with respect to the scale factor $a=1 /(1+z)$, and $\bar{b}_{\text {line }}$ is the luminosity-weighted bias of the tracer, which we consider to be a constant on large scales. $\sigma_{v}$ is the $1 \mathrm{D}$ velocity dispersion within halo mass $M$. Assuming the halos are isothermal, the velocity dispersion can be estimated as

$$
\sigma_{v}^{2}=\frac{G M}{2 r_{\mathrm{vir}}},
$$


where $r_{\text {vir }}$ is the virial radius of the halo.

Combining the projection and RSD effects, the projected $\mathrm{CO}$ power spectrum can be written as

$$
\begin{aligned}
& P_{\mathrm{CO}(1 h)}^{\mathrm{prj}}\left(k_{\mathrm{C} \text { II }}, \mu_{\mathrm{C} \text { II }}\right)=r^{\|}\left(r^{\perp}\right)^{2}\left(1+\frac{f\left(z_{\mathrm{CO}}\right)}{\bar{b}_{\mathrm{CO}}} \mu_{\mathrm{CO}}^{2}\right)^{2} \\
& \quad \times \int_{M_{\min }}^{M_{\max }} d M \frac{d N}{d M}\left[\left(\frac{L_{\text {line }}\left(M, z_{\mathrm{CO}}\right)}{4 \pi D_{L}^{2}\left(z_{\mathrm{CO}}\right)} y_{\mathrm{CO}}(z) D_{A}^{2}\left(z_{\mathrm{CO}}\right)\right)^{2}\right. \\
& \left.\quad \times\left|u\left(k_{\mathrm{CO}} \mid M\right)\right|^{2} e^{-\left(k_{\mathrm{CO}} \sigma_{v} \mu_{\mathrm{CO}}\right)^{2} / 2}\right], \\
& \quad P_{\mathrm{CO}(2 h)}^{\mathrm{prj}}\left(k_{\mathrm{C} \text { II }}, \mu_{\mathrm{C} \text { II }}\right)=r^{\|}\left(r^{\perp}\right)^{2} P_{\mathrm{lin}}\left(k_{\mathrm{CO}}\right)\left(1+\frac{f\left(z_{\mathrm{CO}}\right)}{\bar{b}_{\mathrm{CO}}} \mu_{\mathrm{CO}}^{2}\right)^{2} \\
& \quad \times\left\{\left(\int_{M_{\min }}^{M_{\max }} d M \frac{d N}{d M}\left[\left(\frac{L_{\mathrm{line}}\left(M, z_{\mathrm{CO}}\right)}{4 \pi D_{L}^{2}\left(z_{\mathrm{CO}}\right)} y_{\mathrm{CO}}\left(z_{\mathrm{CO}}\right) D_{A}^{2}\left(z_{\mathrm{CO}}\right)\right)\right)\right.\right. \\
& \left.\left.\quad \times\left|u\left(k_{\mathrm{CO}} \mid M\right)\right| b\left(M, z_{\mathrm{CO}}\right) e^{-\left(k_{\mathrm{CO}} \sigma_{v} \mu_{\mathrm{CO}}\right)^{2} / 2}\right]\right\}^{2} .
\end{aligned}
$$

We define $\mu_{\mathrm{C} \text { II }}$ and $\mu_{\mathrm{CO}}$ to be the cosine angle of the $\boldsymbol{k}_{\mathrm{C} \text { II }}$ and $\boldsymbol{k}_{\mathrm{CO}}$ vectors with respect to the line-of-sight direction, respectively. For the [C II] power spectrum in its own comoving frame, only the RSD effect needs to be considered, so the [C II] 2D power spectrum has the form given by Equations (12) and (13).

To demonstrate the deblending technique, we consider a simple case where the $z_{\mathrm{C} \text { II }}=6$ [C II] line is blended with the brightest $\mathrm{CO}$ line, $\mathrm{CO}(3-2)$ from $z=0.27$, in an IM observing volume. This is our fiducial model. Figure 2 shows the fiducial [C II] and projected $\mathrm{CO}$ power spectra in different $\mu_{\mathrm{C} \text { II }}$ values. We also show power spectra obtained by averaging over $\mu_{\mathrm{C} \text { II }}$, which we called the "ave-prj" power spectrum hereafter. For comparison, we plot the ave-prj power spectra using the SFR$M$ relation in Silva et al. (2015; hereafter S15 model). The aveprj $\mathrm{CO}$ power spectra for other $J$ transitions are shown in Figure 3.

\section{MCMC-BASED PARAMETER INFERENCE}

We construct the $[\mathrm{C} \mathrm{II}]$ and projected $\mathrm{CO}$ power spectra with the CIB model described above, and use them as templates to extract [C II] and CO components in a simulated IM data cube. Specifically, we use the MCMC formalism to extract power spectrum parameters.

\section{1. [C II] and CO Templates}

In summary, the $[\mathrm{C} \mathrm{II}]$ and $\mathrm{CO}$ power spectra are derived based on Equations (12), (13), (15), and (16) ablove. We write the $3 \mathrm{D}$ power spectrum in the following form.

$$
\begin{aligned}
P_{\text {line }}\left(k_{\mathrm{C} \text { II }}, \mu_{\mathrm{C} \text { II }}\right)= & \left(1+\frac{f\left(z_{\text {line }}\right)}{\bar{b}_{\text {line }}} \mu_{\text {line }}^{2}\right)^{2} \\
& \times A_{\text {line }} T_{\text {line }}\left(k_{\mathrm{C} \text { II }}, \mu_{\mathrm{C} \text { II }}\right),
\end{aligned}
$$

where $A_{\text {line }}$ is a constant amplitude of the power spectrum, and $\bar{b}_{\text {line }}$ the luminosity-weighted bias of the emission line. $T\left(k^{\perp}, k^{\|}\right)$is the power spectrum template, given by the sum of the one-halo and two-halo terms in Equations (12), (13), (15), and (16) (without the Kaiser RSD term). Figure 4 shows
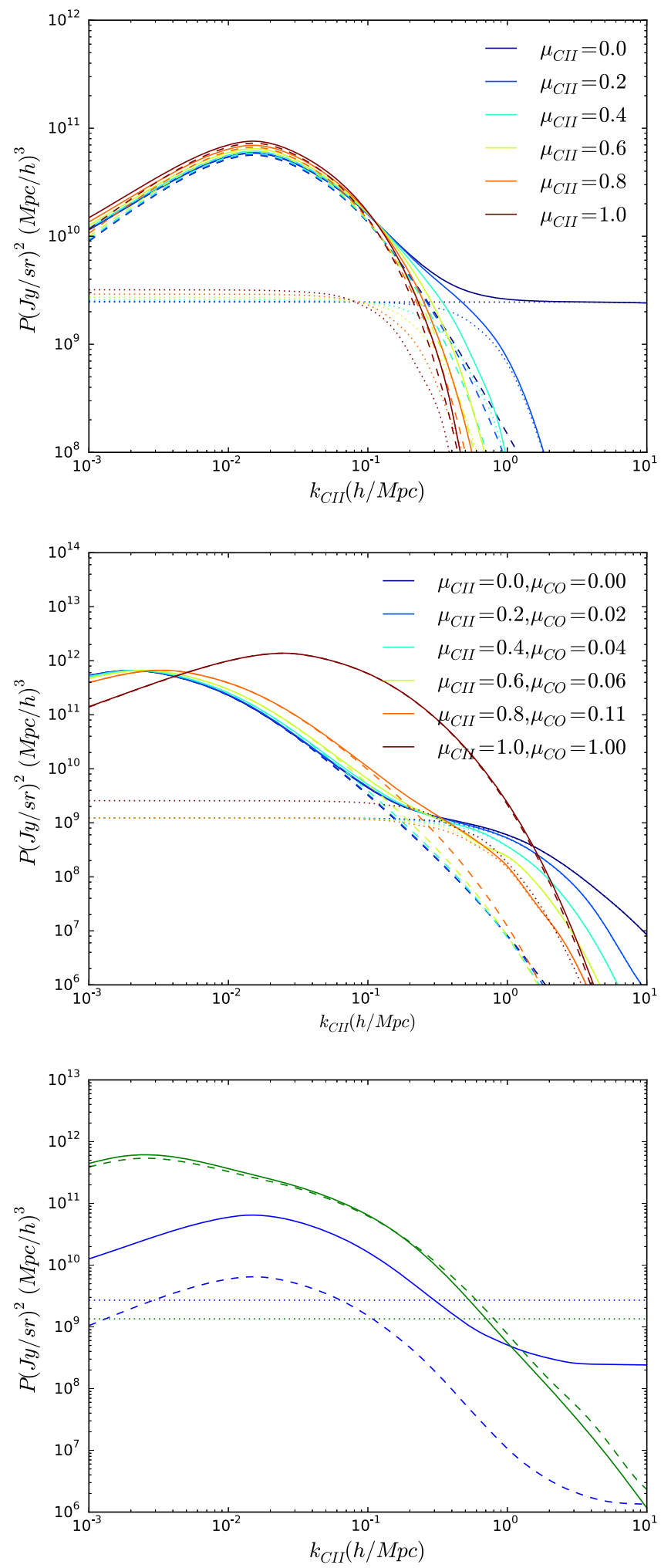

Figure 2. Top: projected [C II] power spectra with different $\mu_{\mathrm{C} \text { II }}$ values in the fiducial model. The dotted lines are one-halo terms, the dash lines are two-halo terms, and the solid lines are the projected total power spectrum $\left(P_{1 h}^{\mathrm{prj}}+P_{2 h}^{\mathrm{prj}}\right)$. Middle: projected $\mathrm{CO}$ power spectra for different $\mu_{\mathrm{C} \text { II }}$ and the corresponding $\mu_{\mathrm{CO}}$. Bottom: the "ave-prj" power spectrum of [C II] (blue) and CO (green) in the fiducial model (solid lines). The dotted lines are the projected shot noise level of the CIB model for [C II] (blue) and CO (green), respectively. The aveprj power spectra from S15 model are shown in dash lines. 


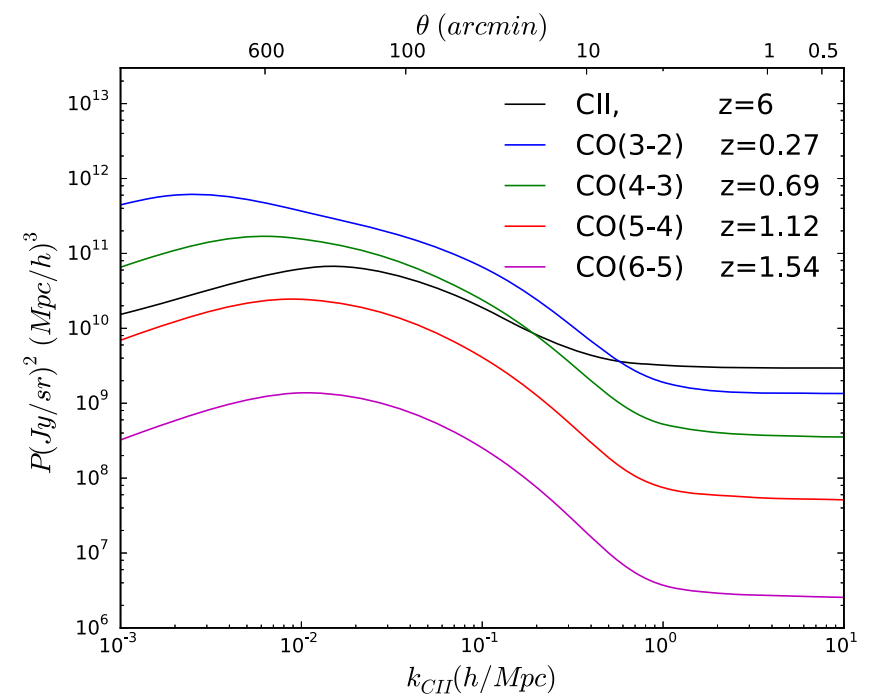

Figure 3. The "ave-prj" power spectra of [C II] and several CO contaminants in the fiducial model. The upper axis is the corresponding observed angular scale given by $\theta=2 \pi / k_{\mathrm{C} \text { II }} \chi(z=6)$.
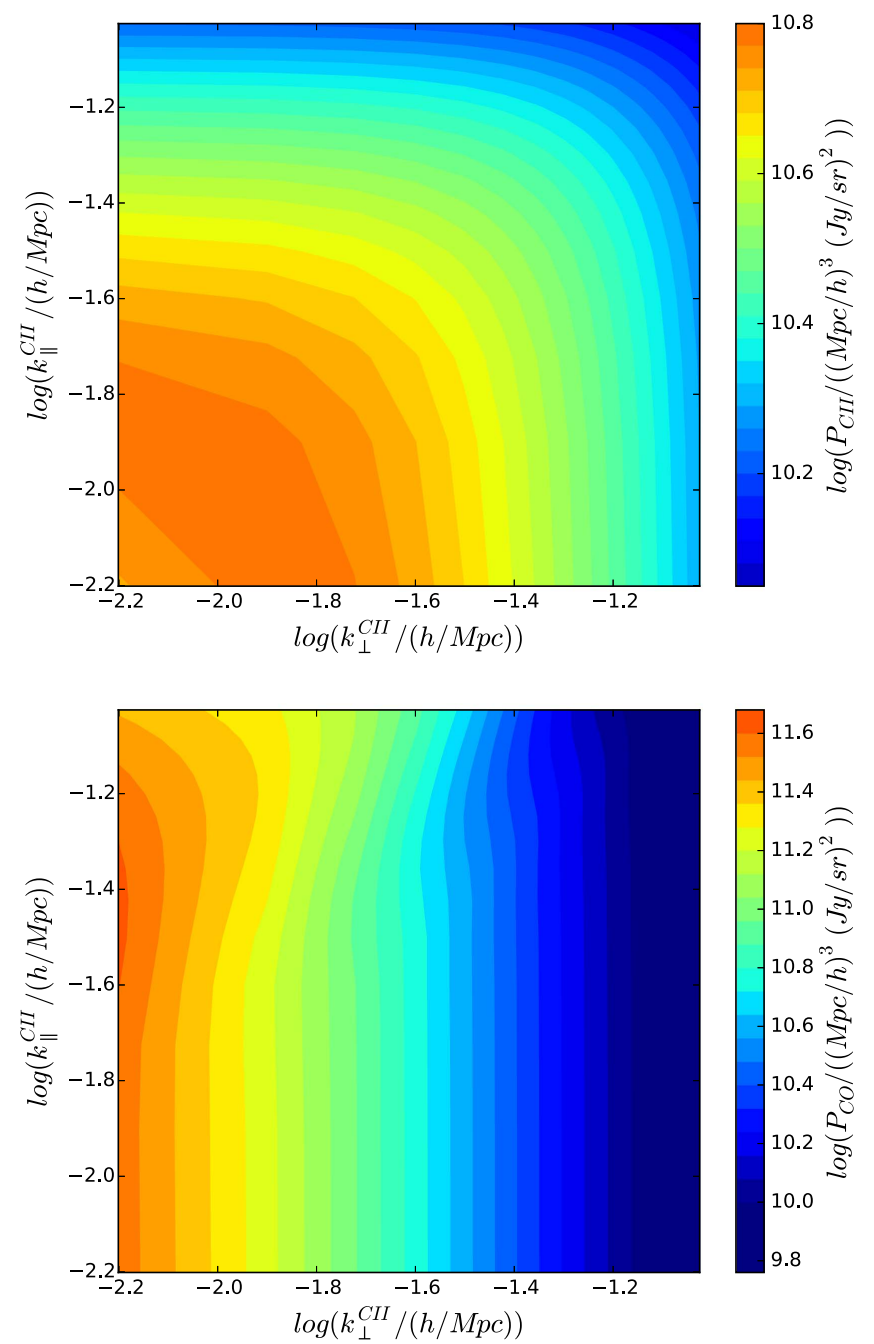

Figure 4. [C II] (top) and $\mathrm{CO}$ (bottom) power spectrum templates with fiducial model in the $k$ range specified in Section 3.2. the $[\mathrm{C} \mathrm{II}]$ and $\mathrm{CO}$ power spectrum templates $T$ for the fiducial model.

We have assumed that the shape of the power spectrum templates $T$ is independent of the luminosity-halo mass relation, so that only an amplitude parameter $A$ is required to describe variations in the model. This assumption is valid on large scales, where the bias parameter is assumed to be scaleindependent and only the linear Kaiser RSD effect is important. The large-scale power spectrum is proportional to $\bar{b}^{2} \bar{I}^{2}$, where $\bar{b}$ and $\bar{I}$ are the luminosity-weighted bias and mean intensity, respectively, and reduces to the linear form: $P(k, \mu)=\left(1+\frac{f(z)}{\bar{b}} \mu^{2}\right)^{2} \bar{b}^{2} \bar{I}^{2} P_{\text {lin }}(k)$. Thus the power spectrum can be easily described by $\bar{b}$ in the Kaiser term (where we assume $f$ is fixed by our chosen cosmology) and an overall amplitude $A$ that is proportional to $\bar{b}^{2} \bar{I}^{2}$. These are the two parameters we fit with MCMC. For this purpose, we restrict ourselves to large scales only. The exact $k$-space range we use is described in Section 3.2. Below we work interchangeably in the $(k, \mu)$ and $\left(k^{\perp}, k^{\|}\right)$space, where $k^{\|}$is the Fourier wavenumber parallel to the light of sight and $k^{\perp}$ perpendicular to it, and $k=\sqrt{\left(k^{\perp}\right)^{2}+\left(k^{\|}\right)^{2}}$ and $\mu=k^{\|} / k$.

\section{2. $k$-space Range}

We consider a cubic survey size with a spatial dimension of $10 \times 10 \mathrm{deg}^{2}$. At $z=6$, this corresponds to a linear comoving scale of $952 \mathrm{Mpc} / h$; assuming the same comoving scale in the light-of-sight direction, we have an IM survey volume of $(952)^{3}(\mathrm{Mpc} / h)^{3}$. We restrict our analysis to large scales only, with $k_{\mathrm{CII}}<0.1 \mathrm{~h} \mathrm{Mpc}^{-1}$. For [C II] at $z=6$, the smallest accessible $k^{\perp}$ mode is $k_{\mathrm{CII}}^{\min }=6.3 \times 10^{-3} h \mathrm{Mpc}^{-1}$, and we choose the maximum to be $k_{\mathrm{CII}}^{\max }=9.5 \times 10^{-2} h \mathrm{Mpc}^{-1}$. We consider the same limit for $k^{\|}$. Thus we have $15 \times 15 k$ space pixels in the mock data and the templates.

\subsection{Mock Observed Power Spectrum Construction}

We generate a mock data power spectrum $P_{\text {data }}$, which consists of the redshifted [C II] and $\mathrm{CO}$ power spectra, and a noise contribution $\delta P$ :

$$
\begin{aligned}
P_{\text {data }}\left(k_{\mathrm{C} \text { II }}, \mu_{\mathrm{C} \text { II }}\right)= & P_{\mathrm{C}_{\text {II }}}\left(k_{\mathrm{C} \text { II }}, \mu_{\mathrm{C} \text { III }}\right) \\
& +P_{\mathrm{CO}}\left(k_{\mathrm{C}_{\text {II }}}, \mu_{\mathrm{C} \text { II }}\right)+\delta P\left(k_{\mathrm{C} \text { II }}, \mu_{\mathrm{C} \text { II }}\right),
\end{aligned}
$$

where $P_{\mathrm{C} \text { II }}$ and $P_{\mathrm{CO}}$ are described in Equation (17). $\delta P$ are random values that account for power spectrum noise. For each $k$-space pixel, $\delta P$ is drawn from a Gaussian distribution with zero mean and variance $\sigma_{P}^{2}\left(k_{\mathrm{C} \mathrm{II}}, \mu_{\mathrm{C} \text { II }}\right)$ :

$$
\begin{aligned}
\sigma_{P}\left(k_{\mathrm{C} \text { II }}, \mu_{\mathrm{C} \text { II }}\right)= & \frac{1}{\sqrt{N\left(k_{\mathrm{C} \text { II }}, \mu_{\mathrm{C} \mathrm{II}}\right)}} \\
& \times\left(P_{\text {data }}\left(k_{\mathrm{C} \text { II }}, k_{\|}\right)+P_{\mathrm{sh}}^{\mathrm{C} \mathrm{II}}+P_{\mathrm{sh}}^{\mathrm{CO}}+P_{n}\right),
\end{aligned}
$$

where the $P_{\text {data }}$ term accounts for cosmic variance, $P_{\mathrm{sh}}^{\mathrm{C} \mathrm{II}}$ and $P_{\mathrm{sh}}^{\mathrm{CO}}$ are the shot noise contributions (see Equation (3)), and $P_{n}$ is the instrument noise power spectrum. In this work, we assume $P_{n}=1.77 \times 10^{9}\left(\mathrm{Jy} \mathrm{sr}^{-1}\right)^{2}(\mathrm{Mpc} / h)^{3}$, which is consistent with the thermal noise level of current generation of planned [C II] IM experiments, such as the TIME-pilot (Crites et al. 2014); though, TIME-pilot plans a smaller survey volume 
than considered here. $N\left(k_{\mathrm{C}}\right.$ II,$\left.\mu_{\mathrm{C} \text { II }}\right)$ is the number of pixels in each $k$ bin.

We do not include the shot noise and instrument white noise contributions in the template and in the mock data, since we assume they can be measured and subtracted before the template fitting process. In a real experiment, these constant noises are the dominant "signals" at high- $k$, so we are able to infer the noise level from the high $k$ modes and subtract them from the data.

\subsection{MCMC Implementation}

For a given set of parameters $\left\{A_{\mathrm{C} \text { II }}, A_{\mathrm{CO}}, b_{\mathrm{C}}\right.$ II,$\left.b_{\mathrm{CO}}\right\}$, the model power spectrum $P_{\text {model }}$ is given by

$$
\begin{aligned}
& P_{\text {model }}\left(k_{\mathrm{C} \text { II }}, \mu_{\mathrm{C}_{\text {II }}}\right) \\
& =\left(1+\frac{f\left(z_{\mathrm{C} \text { II }}\right)}{\bar{b}_{\mathrm{C} \text { II }}} \mu_{\mathrm{C} \text { II }}^{2}\right)^{2} A_{\mathrm{C} \text { II }} T_{\mathrm{C}_{\text {II }}}\left(k_{\mathrm{C} \text { II }}, \mu_{\mathrm{C}_{\text {II }}}\right) \\
& \quad+\left(1+\frac{f\left(z_{\mathrm{CO}}\right)}{\bar{b}_{\mathrm{CO}}} \mu_{\mathrm{CO}}^{2}\right)^{2} A_{\mathrm{CO}} T_{\mathrm{CO}}\left(k_{\mathrm{C} \text { II }}, \mu_{\mathrm{C} \text { II }}\right) .
\end{aligned}
$$

The log-likelihood expression is

$$
\begin{aligned}
\ln \mathcal{L}= & -\frac{1}{2} \sum_{k}\left\{\frac{\left(P_{\text {data }}\left(k_{\mathrm{C} \text { II }}, \mu_{\mathrm{C} \text { II }}\right)-P_{\text {model }}\left(k_{\mathrm{C} \text { II }}, \mu_{\mathrm{C} \text { II }}\right)\right)^{2}}{\left(\sigma_{P}\left(k_{\mathrm{C} \text { II }}, \mu_{\mathrm{C} \text { II }}\right)\right)^{2}}\right. \\
& \left.+\ln \left[2 \pi\left(\sigma_{P}\left(k_{\mathrm{C} \text { II }}, \mu_{\mathrm{C}_{\text {II }}}\right)\right)^{2}\right]\right\} .
\end{aligned}
$$

We set flat priors for $A_{\mathrm{C} \text { II }}$ and $A_{\mathrm{CO}}$ in the range of $\left[10^{-6}, 10^{6}\right]$ (fiducial value $=1$ ), and flat priors for $\bar{b}_{\mathrm{C} \text { II }}$ and $\bar{b}_{\mathrm{CO}}$ between $[0.1,20]$, and zero otherwise.

We use Python package emcee v2.1.0 (Foreman-Mackey et al. 2013) to perform the MCMC analysis. We use an ensemble of 1000 walkers taking 1000 steps after 1000 burn-in steps.

\section{RESULTS}

\subsection{Fiducial Model}

Figure 5 shows the posterior distribution of the four parameters in the fiducial case, where $\left\{A_{\mathrm{C} \text { II }}, A_{\mathrm{CO}}, \bar{b}_{\mathrm{C} \text { II }}, \bar{b}_{\mathrm{CO}}\right\}=\{1,1,7.20,1.48\}$. For each point in the four-dimensional parameter space, we construct an aveprj power spectrum by averaging over $\mu_{\mathrm{C}}$ II of a $3 \mathrm{D}$ power spectrum specified by these parameters. Instead of examining the amplitude parameters $A_{\mathrm{C} \text { II }}$ and $A_{\mathrm{CO}}$, we use the ave-prj power spectra to compare the input and output amplitudes.

Next, we consider more general cases by changing the input amplitudes $A_{\mathrm{C} \text { II }}$ and $A_{\mathrm{CO}}$ in the mock data. The [C II] and $\mathrm{CO}$ shot noise levels also vary accordingly with the clustering amplitudes. We first fix $A_{\mathrm{C} \text { II }}=1$ and run MCMC with $A_{\mathrm{CO}}=[0.01,0.1,1,10,100]$. Figure 6 (left) shows the $68 \%$ confidence interval of ave-prj [C II] power spectrum amplitude relative to the input, $P_{\mathrm{C} \text { II }}^{\text {true }}$. For comparison, we also calculate the amplitude of the noise power spectrum $\sigma_{P}$ (see Equation (19)) relative to the best-fit value of the ave-prj [C II] power spectrum $P_{\mathrm{C} \text { II }}^{\text {best }}$. We define a quantity $A_{\sigma}$ to be the median value of the ratio $\sigma_{P} / P_{\mathrm{C} \text { II }}^{\text {best }}$ over the $15 \times 15 k$-space pixels. $A_{\sigma}$ serves as an indicator of the available information content level, which we discuss further in Section 5.4. The S/ $\mathrm{N} \equiv P_{\mathrm{C} \text { II }} / \Delta P_{\mathrm{C} \text { II }}$ of [C II] ave-prj power spectrum is shown in Figure 6 (right), where $\Delta P_{\mathrm{C} \text { II }}$ is the standard deviation of the

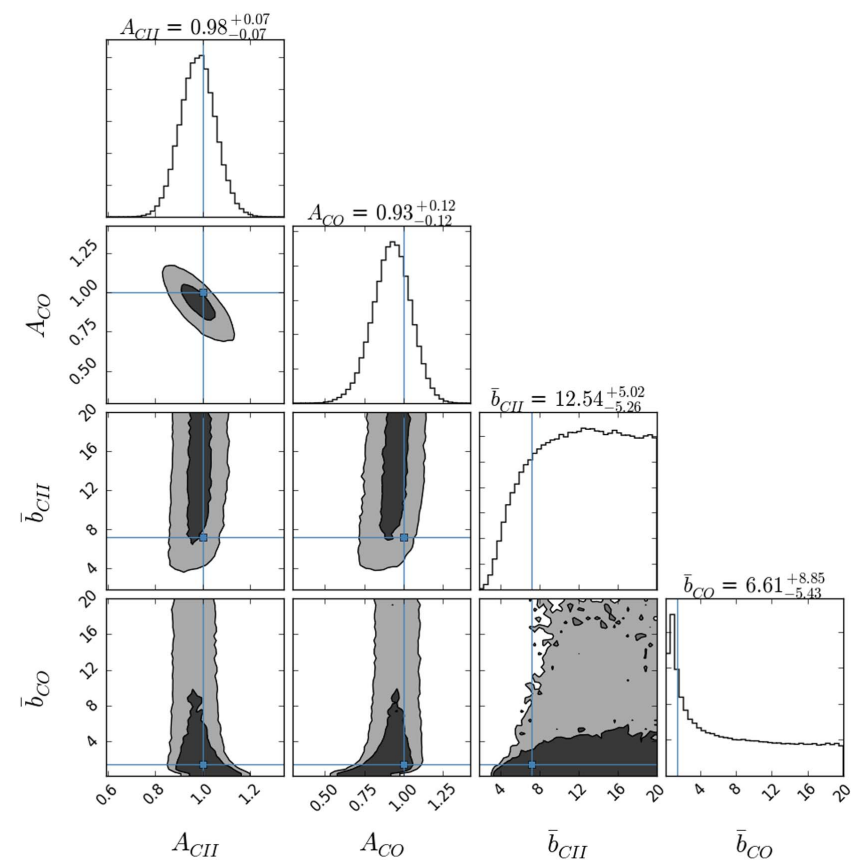

Figure 5. MCMC posterior distribution of the fiducial case. The contours marked the $68 \%$ and $95 \%$ confidence interval in the parameter space. Crosshairs indicate the input value of the data: $\left\{A_{\mathrm{C} \text { II }}=1, A_{\mathrm{CO}}=1, \bar{b}_{\mathrm{C} \text { II }}=7.20, \bar{b}_{\mathrm{CO}}=1.48\right\}$.

ave-prj power spectrum amplitudes given by MCMC. We then fix $A_{\mathrm{CO}}=1$ and set $A_{\mathrm{C} \text { II }}=[0.01,0.1,1,10,100]$ to repeat the exercise. The results are shown in Figure 7.

In the three cases with high $[\mathrm{C} \mathrm{II}]$ to $\mathrm{CO}$ ratios, where $A_{\sigma}<1$, the mean of the ave-prj [C II] power spectrum can be reproduced within $10 \%$ deviation from the true value, with $\mathrm{S} /$ $\mathrm{N}>10$ in both tests. Not all of the true ave-prj [C II] power spectrum amplitudes, however, fall in the $68 \%$ interval of the MCMC distributions. To understand this inconsistency, we run 100 realizations of mock data with the fiducial case $\left(A_{\mathrm{C} \text { II }}=A_{\mathrm{CO}}=1\right)$. We find that in 63 of the 100 runs, the ave-prj $P_{\mathrm{C} \text { II }}^{\text {true }}$ does fall in the $68 \%$ interval of the MCMC distribution. This suggests that parameter degeneracy may exist in the fitting, so that some of the fitted amplitudes deviate slightly from the true values. This issue might be resolved by adopting tighter constraints on the input $\bar{b}_{\mathrm{C}}$ and $\bar{b}_{\mathrm{CO}}$. We will investigate this degeneracy in future work.

For the rest, where $A_{\sigma}>1$, the $\mathrm{S} / \mathrm{N}$ given by MCMC degrade to less than 10 and one of the mean values is biased by a factor of four; we find $A_{\sigma}$ to be a good indicator in determining the reliability of the fitting.

Figure 8 shows the ave-prj power spectrum for all nine combinations of input $A_{\mathrm{C} \text { II }}$ and $A_{\mathrm{CO}}$ we discuss above. Reproducing the best-fit mock data with the four parameters $\left\{A_{\mathrm{C} \text { II }}, A_{\mathrm{CO}}, \bar{b}_{\mathrm{C} \text { II }}, \bar{b}_{\mathrm{CO}}\right\}$ and comparing with the input mock data, we calculate the $\chi^{2}$ in each cases, and the probability to exceed (PTE) the observed value if MCMC gives correct parameters. The PTE values are also shown in Figure 8.

\subsection{S/N Dependence on Noise Level}

We assume the instrument noise can be subtracted from the data power spectrum before template fitting, but it still contributes a $k$-space noise in Equation (19). Here we investigate the effect of instrument noise level $P_{n}$ on the fitted results. We again fix input $A_{\mathrm{C} \text { II }}=1$ and $A_{\mathrm{CO}}=[0.01,0.1,1$, 

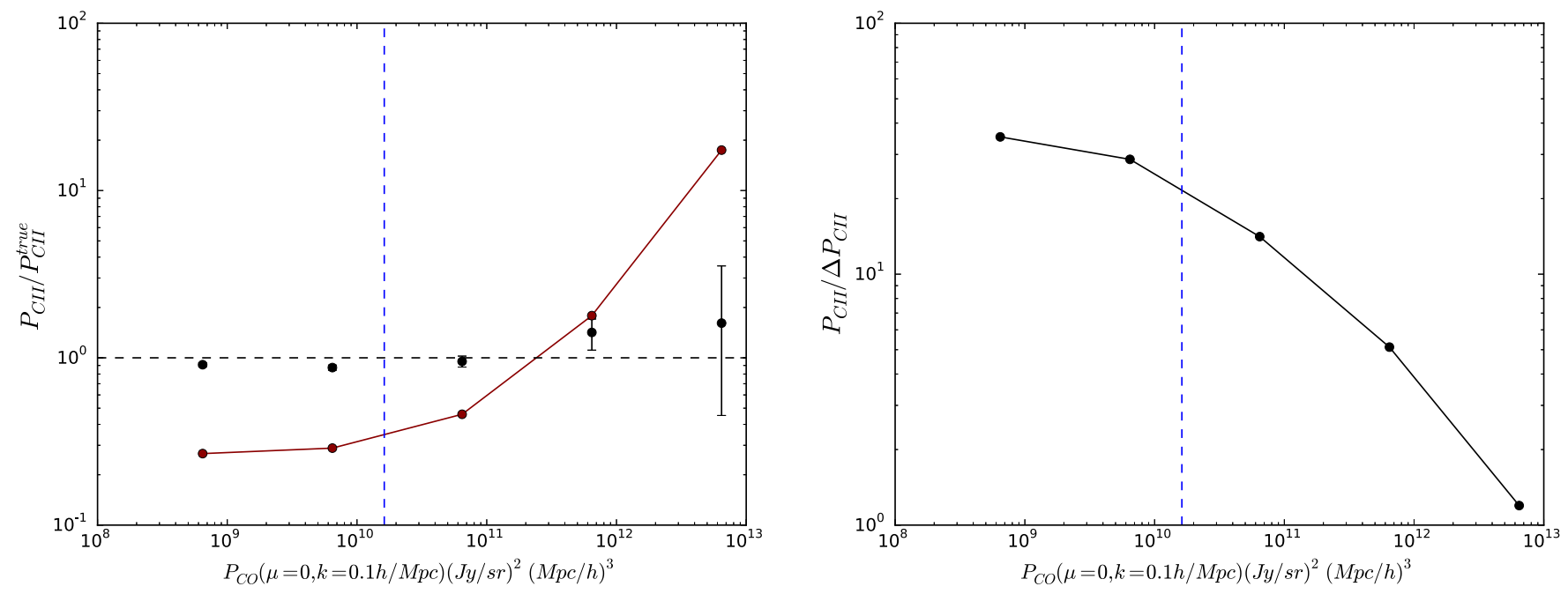

Figure 6. Left: the ave-prj power spectrum amplitude from MCMC relative to the input value in the mock data. The inputs are $A_{\mathrm{C} \text { II }}=1$, and $A_{\mathrm{CO}}=[0.01,0.1,1,10$, 100] from left to right. The $x$-axis is expressed in the $\mathrm{CO}$ ave-prj power spectrum at $k_{\mathrm{C}}$ II $=0.1 \mathrm{~h} \mathrm{Mpc}^{-1}$ for better comparison. The blue dash line indicates the input [C II] prj-ave power spectrum at $k_{\mathrm{C} \text { II }}=0.1 \mathrm{~h} \mathrm{Mpc}^{-1}$. The error bars are the $68 \%$ confident interval given by MCMC. The dark red line indicates the $A_{\sigma}$ values (see the text). Right: $\mathrm{S} / \mathrm{N}$ of the ave-prj [C II] power spectrum amplitude given by MCMC.

$10,100]$, and run a series of cases by changing $P_{n}$ to be $[0.1,1$, $10, \quad 100,1000]$ times the initial $P_{n}$ value $\left(P_{n, i}=1.77 \times 10^{9}\left(\mathrm{Jy} \mathrm{sr}^{-1}\right)^{2}(\mathrm{Mpc} / h)^{3}\right)$. The results are shown in Figure 9. For comparison, We also calculate the theoretical $\mathrm{S} / \mathrm{N}$ on the [C II] power spectrum, which can be expressed as

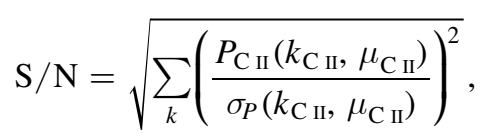

where $\sigma_{P}$ is given by Equation (19), and summing over all $k$ space pixels.

Note that all of the highest $P_{n}$ cases show biased results, and some of the large $P_{n}$ cases also give amplitudes that deviate significantly from the input values. This may be indicative that given the survey size we consider in this work, $P_{n} \approx 10^{10}\left(\mathrm{Jy} \mathrm{sr}^{-1}\right)^{2}(\mathrm{Mpc} / h)^{3}$ is the maximum allowed instrument noise for detecting the CIB-based [C II] signals. We will conduct a more detailed investigation in future work.

\subsection{Multiple Foreground Lines}

So far, we have only considered the brightest foreground $\mathrm{CO}$ (3-2) line at $z=0.27$ to the $z=6[\mathrm{C} \mathrm{II}]$ signal. Here we extend the technique to incorporate two more foreground lines: $\mathrm{CO}(4-$ 3 ) from $z=0.69$ and $\mathrm{CO}(5-4)$ from $z=1.12$. The power spectra of $J>5$ transition lines are more than two orders of magnitude lower than the expected [C II] signal, and we do not consider them in this paper.

The extra $\mathrm{CO}$ lines can be incorporated by extending Equations (18) and (20) with two more CO terms, which introduce two more amplitudes and bias factors in the MCMC procedure. Thus we now fit the mock data with eight parameters in the same $k$-space defined before. We set all the input amplitude to be unity, so the mock data is consistent with the CIB model prediction.

The result is shown in Figure 10. The [C II] ave-prj amplitude given by MCMC has an $\mathrm{S} / \mathrm{N}=4.12$, and the input [C II] ave-prj amplitude slightly falls outside the $68 \%$ confidence interval. The technique appears to be valid even in the presence of multiple foreground lines that overwhelm the
[C II] signals in the $k$ range we consider, though at the cost of $\mathrm{S} / \mathrm{N}$ for the extracted $[\mathrm{C} \mathrm{II}]$ signal.

\section{DISCUSSION}

\subsection{Model Dependence}

In reality, if the templates do not perfectly describe the true signal intensity field, there will be amplitude and shape discrepancies between the template and true signal power spectra. The overall amplitude discrepancy can be fully absorbed by the amplitude parameters $A_{\mathrm{C} \text { II }}$ and $A_{\mathrm{CO}}$ in our fitting process. Power spectrum shape difference may arise from different assumed $L-M$ relations, but since we restrict our fitting to the large-scale clustering terms, the procedure is not susceptible to incorrect model assumptions. As a sanity check, we use templates generated from the S15 model to fit the same set of mock data discussed in Section 4.1, which are constructed with the CIB model. Figure 11 shows the ave-prj power spectrum from fitting the S15 template to the CIB model mock data. The results for all the nine scenarios considered before are also consistent with the results presented in Section 4.1. The template fitting technique is robust against model uncertainties as long as only large-scale information is considered.

Conversely, this model-independent property implies that the technique constrains only the overall amplitude of the power spectrum and is not sensitive to different $L-M$ relation scenarios.

\subsection{Model Uncertainties}

While [C II] and CO modeling uncertainties do not bias the template fitting results, they affect the quality of the fit. In Section 2, we show that the CIB model considered in this work gives rise to a high [C II] power and a large bias factor. Our modeling of the different $\mathrm{CO} J$ rotational lines also determines the relative amplitudes of interlopers and the severity of contamination.

We have therefore conducted a series of tests with different input $[\mathrm{C} \mathrm{II}]$ and $\mathrm{CO}$ amplitudes and varying noise levels to account for the uncertainties. We find that if the true [C II] 

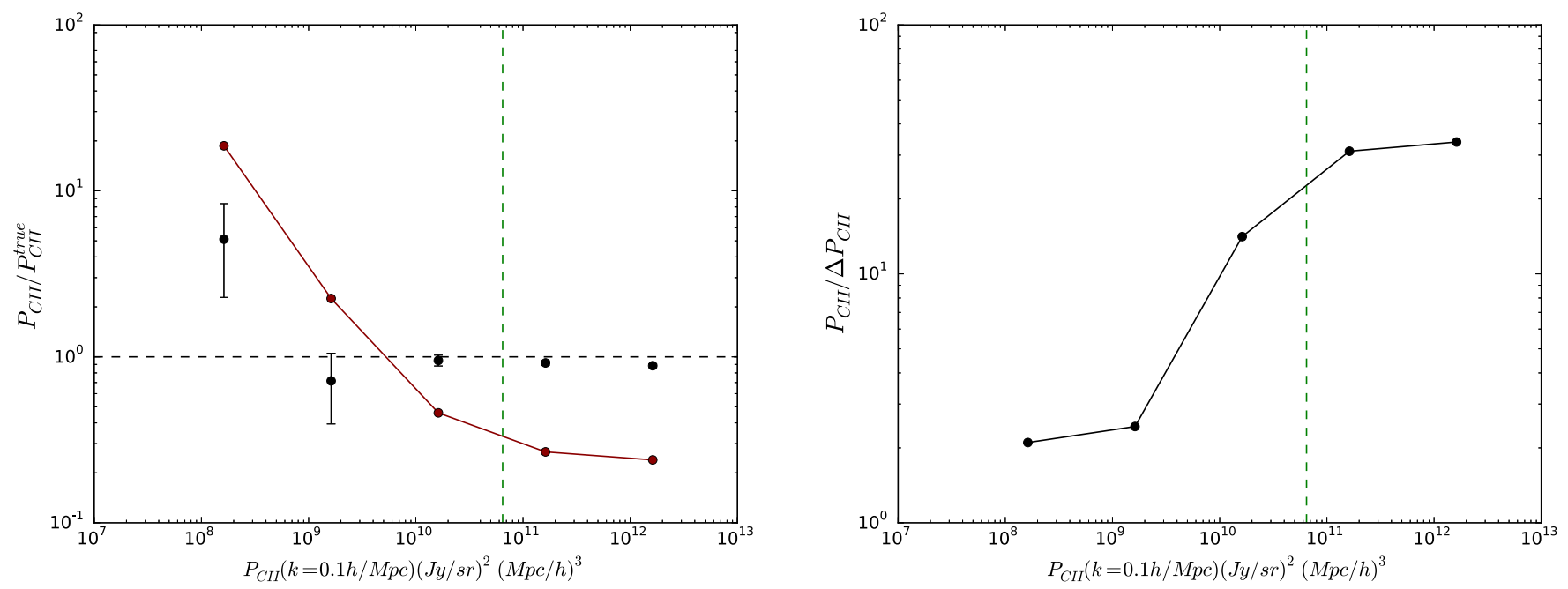

Figure 7. Left: the ave-prj power spectrum amplitude from MCMC relative to the input value in the mock data. The inputs are $A_{\mathrm{CO}}=1$, and $A_{\mathrm{C} \text { II }}=[0.01,0.1,1,10$, 100] from left to right. The $x$-axis is expressed in the [C II] ave-prj power spectrum at $k_{\mathrm{C} \text { II }}=0.1 h \mathrm{Mpc}^{-1}$ for better comparison. The green dashed line indicates the input CO prj-ave power spectrum at $k_{\mathrm{C} \text { II }}=0.1 \mathrm{~h} \mathrm{Mpc}^{-1}$. The error bars are the $68 \%$ confident interval given by MCMC. The dark red line indicates the $A_{\sigma}$ values (see the text). Right: $\mathrm{S} / \mathrm{N}$ of the ave-prj [C II] power spectrum amplitude given by MCMC.

power is 10 times smaller than the fiducial CIB amplitude (second-left point in Figure 7), for instance, $A_{\sigma}$ goes above unity, which implies a non-detection, contrary to the more optimistic fiducial case.

To get a sense of how much the results vary with the assumed models, we run simulations with mock data and templates generated from the S15 model. Compared to the CIB model, the S15 model has slightly lower bias values for both [C II] and $\mathrm{CO}$ lines and a similar $\mathrm{CO}$ power spectrum amplitude, while the [C II] power spectrum amplitdue is lower by about one order of magnitude (see the bottom panel of Figure 2). Figure 12 shows the MCMC result of the S15 model. The $\mathrm{S} / \mathrm{N}$ the on ave-prj [C II] power spectrum in this case is 4.5. For comparison, the fiducial CIB model has an $\mathrm{S} / \mathrm{N}$ of $\approx 14$. We find that the fitted [C II] power spectrum $\mathrm{S} / \mathrm{N}$ depends sensitively on its amplitude, when overwhlemed by the $\mathrm{CO}$ foregrounds.

In a similar work by Lidz \& Taylor (2016, in prep), the authors modeled the power spectrum with an SFR that follows the Schechter functional form (Schechter 1976), and the [C II] power spectrum they derived at $z=7$ was about one order of magnitude smaller than our fiducial $z=6$ prediction. Their assumed survey volume was about 5.6 times smaller than considered here. As a result, the [C II] amplitude constraints in their study using the Fisher matrix formalism appeared less optimistic than ours, but the two results are broadly in agreement. In reality, the feasibility of this deblending method will be highly dependent on the assumed survey geometry and $\mathrm{S} / \mathrm{N}$ strengths. We will leave a more realistic [C II] and $\mathrm{CO}$ power spectrum modeling to future work, while cautioning that built-in detection margins are important for planned experiments.

\subsection{Continuum Foreground}

In this paper, we focus on the de-confusion technique that handles spectral line foregrounds. For completeness, we note that at the frequency range of interest, $\sim 200-300 \mathrm{GHz}$, continuum foregrounds are non-negligible and generally stronger than line foregrounds. For our purpose, the main continuum foregrounds include the cosmic microwave background and CIB radiations; the two contribute comparably. Silva et al. (2015) estimates that the dust continuum emission is of the order of $10^{5} \mathrm{Jy} \mathrm{sr}^{-1}$, which is two or three orders of magnitude (depending on model) higher than the [C II] intensity considered in this work. However, since the continuum signals are expected to be spectrally smooth, they dominate the low $k^{\|}$modes in power spectrum space. We therefore expect to be able to mitigate the effect by removing or avoiding the one or two lowest $k^{\|}$modes before template fitting. This is the same technique envoked in the well-studied field of $21 \mathrm{~cm}$ IM (e.g., Liu \& Tegmark 2011; Parsons et al. 2012; Switzer et al. 2015) and implemented on IM data (e.g., Paciga et al. 2013; Switzer et al. 2013; Ali et al. 2015; Keating et al. 2015). To test the impact of continuum foreground mode removal, we run a fiducial MCMC fit, same as the case in Section 4.1 but removing the lowest $k^{\|}$modes. The results are shown in Figure 13. The $\mathrm{S} / \mathrm{N}$ on the $[\mathrm{C}$ II] power spectrum remains nearly the same as the fiducial one (Figure 5). This simple test demonstrates that continuum foregrounds are unlikely to be a major concern, but we note that subtler issues, such as the exact number of $k^{\|}$modes to be removed and the amount of residual continuum in the data, need to be quantified and further tested in the future.

\subsection{Detection Limit}

In Section 4.1, we define $A_{\sigma}$ to be the median value of $\sigma_{P} / P_{\mathrm{C} \text { II }}^{\text {best }}$ and use it as an indicator of the available information content level; when $A_{\sigma}<1$, or $\sigma_{P}<P_{\mathrm{C} \text { II }}^{\text {best }}$, we recover an unbiased estimate of $P_{\mathrm{C} \text { II }}^{\text {true }}$. Indeed, in Section 4 , the scenarios with high $\mathrm{CO}$ to [C II] amplitudes result in biased extracted [C II] power spectrum amplitudes, however, the extracted amplitudes are all below the noise level (i.e., $A_{\sigma}<1$ ). This suggests that the $k$-space noise level is a good indicator of the information content and sets the limit for extracting [C II] signals from the data, below which the MCMC likely returns biased results. In reality, $\sigma_{P}$ and $P_{\mathrm{C} \text { II }}^{\text {best }}$ are quantities that can be directly inferred from real data, and serve to evaluate the reliability of the extracted results. 

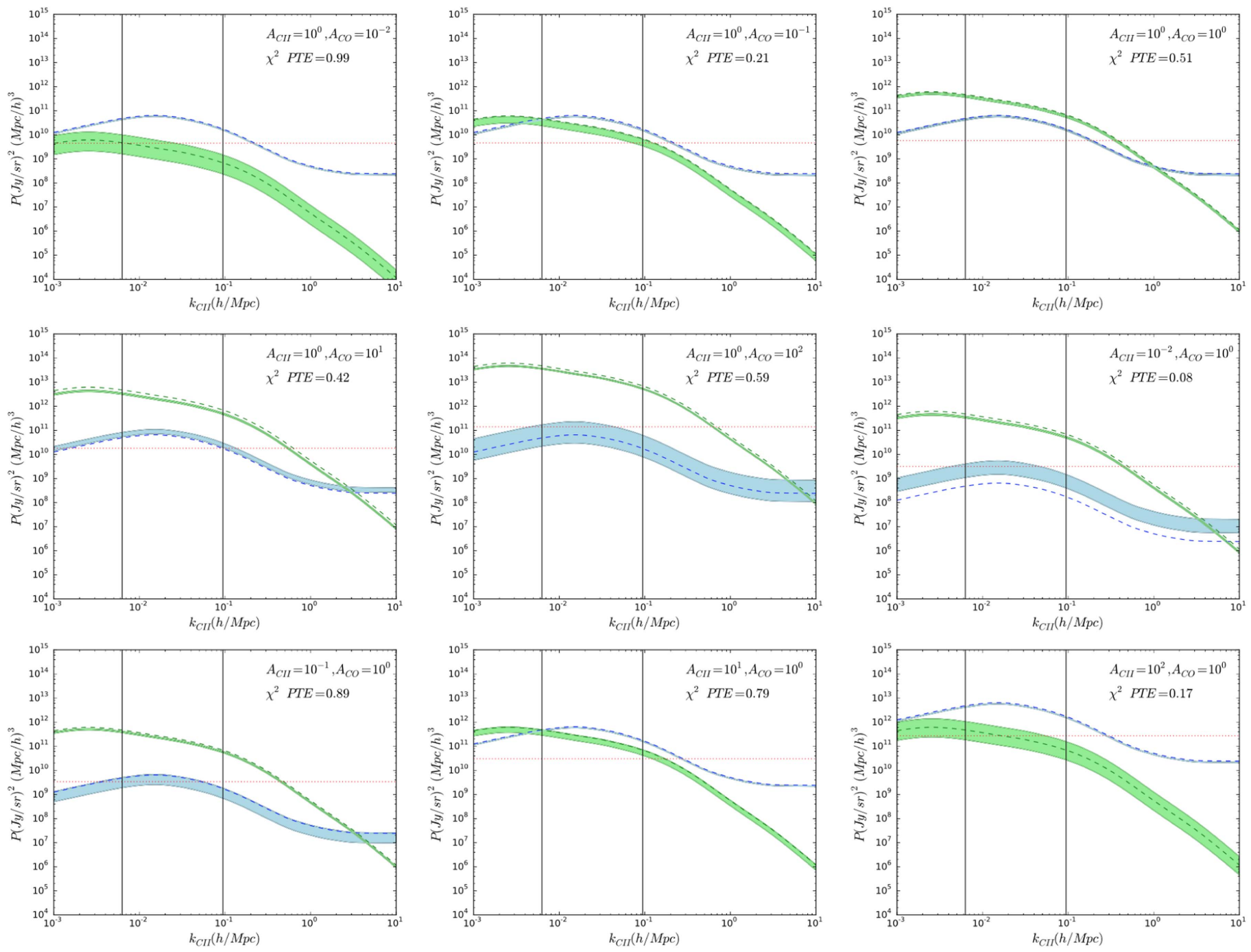

Figure 8. The ave-prj power spectrum with different combinations of input $A_{\mathrm{C} \text { II }}$ and $A_{\mathrm{CO}}$. The blue and green regions are the $68 \%$ confidence interval of [C II] and $\mathrm{CO}$ ave-prj power spectrum respectively. The dashed lines are the input [C II](blue) and $\mathrm{CO}$ (green) ave-prj power spectrum of the mock data. The red dotted line indicates $P_{\mathrm{sh}}^{\mathrm{C} \text { II }}+P_{\mathrm{sh}}^{\mathrm{CO}}+P_{n}$, which is the constant power spectrum in the data that contribute to the noise in $k$ space (see Equation (19)). The two vertical black lines are $k_{\mathrm{C} \text { II }}^{\text {min }}$ and $k_{\mathrm{C} \text { II }}^{\max }$ marking the $k$ space region we use in template fitting. The $\chi^{2}$ PTE for each MCMC fit is also provided.

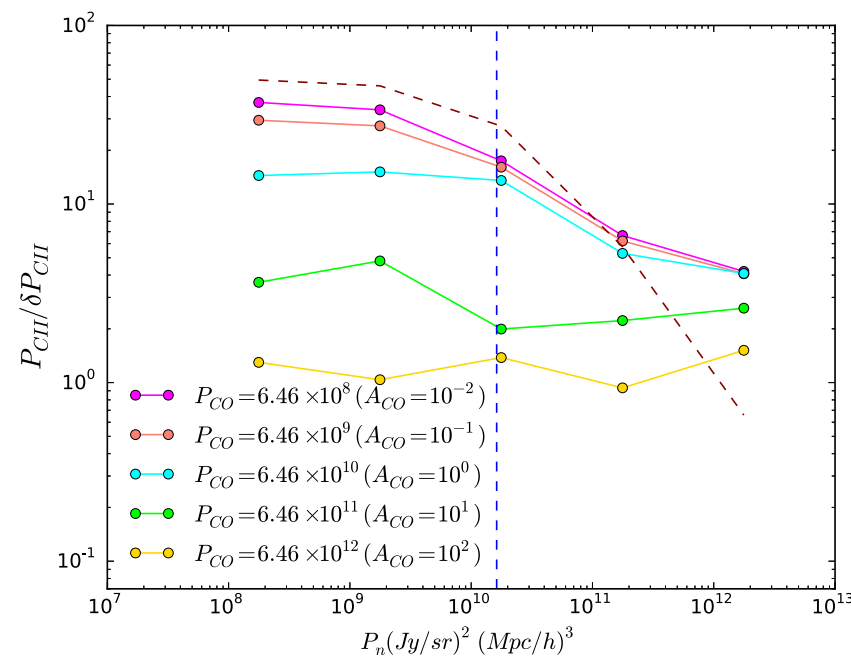

Figure 9. $\mathrm{S} / \mathrm{N}$ of the ave-prj [C II] power spectrum amplitude as a function of $P_{n}$ and $P_{\mathrm{CO}}$. The $P_{\mathrm{CO}}$ written on the top right corner is the ave-prj power spectrum at $k=0.1 h \mathrm{Mpc}^{-1}$. The blue dashed line marks the [C II] ave-prj power spectrum at $k=0.1 \mathrm{~h} \mathrm{Mpc}^{-1}$. The dark red dashed line is the $\mathrm{S} /$ $\mathrm{N}$ derived from mode counting (see the text).

\subsection{Constraint on Bias}

In our template fitting procedure, the bias factors are loosely constrained by MCMC (see Figures 5 and 10). This can be understood by looking at the Kaiser RSD term: $\left(\frac{f(z))}{\bar{b}_{\text {line }}} \mu^{2}\right)^{2}$, where $f \approx 1$, the bias is usually between 1 and 10 , and $\mu^{2}$ is a value between 0 and 1 . Thus $\frac{f(z))}{\bar{b}_{\text {line }}} \mu^{2}$ is usually smaller than unity, sub-dominent to the overall amplitude change of the power spectrum, which is absorbed in the $A$ parameter. In addition, the MCMC constraint on $\bar{b}_{\mathrm{CO}}$ is weaker than on $\bar{b}_{\mathrm{CO}}$. This is due to the projection effect that makes the projected $\mu_{\mathrm{CO}}$ very small for most of the $k$-space pixels (see Figure 2), which are then not sensitive to the Kaiser effect.

The CIB model gives a high $\bar{b}_{\mathrm{C} \text { II }}$ value that makes the Kaiser effect too small to be detected. This can be seen from the histogram of $\bar{b}_{\mathrm{C}}$ II given by MCMC, which extends to large values that correspond to a non-detection of the Kaiser RSD effect. To test the ability to extract bias in our procedure, we run the fiducial case and set $\bar{b}_{\mathrm{C} \text { II }}$ to be the same as the fiducial value of $\bar{b}_{\mathrm{CO}}=1.48$. In this case, the $\bar{b}_{\mathrm{C} \text { II }}$ can be constrained by MCMC with an $\mathrm{S} / \mathrm{N}>5$; while the $\bar{b}_{\mathrm{CO}}$ is still 


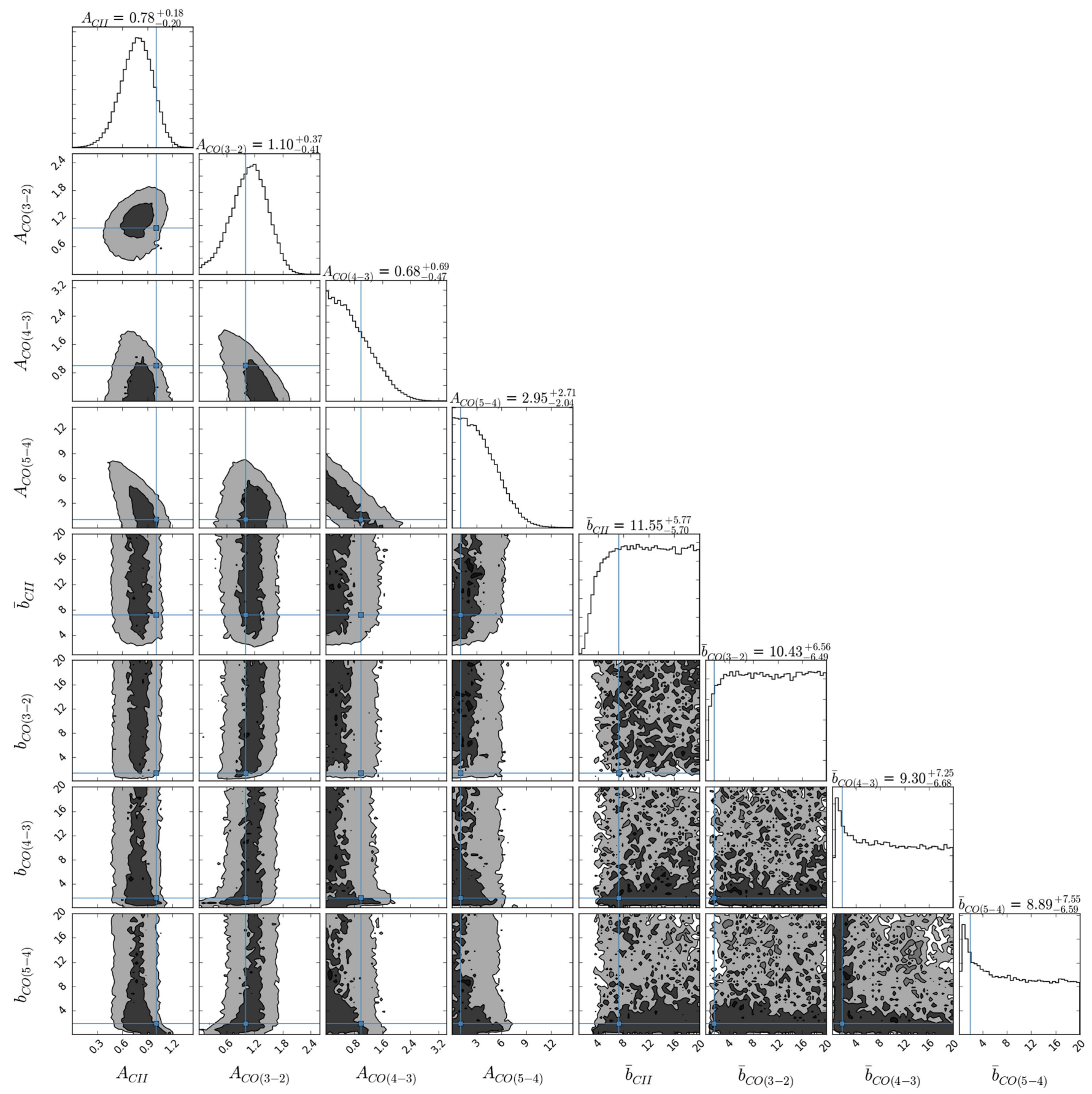

Figure 10. MCMC posterior distribution on the parameter space with three CO foreground lines. The contours marked the $68 \%$ and $95 \%$ confidence interval in the parameter space. Crosshairs indicate the input value of the data: $\left\{A_{\mathrm{C} \text { II }}=1, A_{\mathrm{CO}(3-2)}=1, A_{\mathrm{CO}(4-3)}=1, A_{\mathrm{CO}(5-4)}=1, \bar{b}_{\mathrm{C} \text { II }}=7.20, \bar{b}_{\mathrm{CO}(3-2)}=1.48\right.$, $\left.\bar{b}_{\mathrm{CO}(4-3)}=1.70, \bar{b}_{\mathrm{CO}(5-4)}=1.94\right\}$.

unconstrained due to the projection effect described above. Therefore, if $\bar{b}_{\mathrm{C} \text { II }}$ in reality is smaller than the value we considered in this work, we might be able to better constrain $\bar{b}_{\mathrm{CII}}$. We will investigate strategies to better extract the bias information in future work.

\section{CONCLUSION}

We demonstrate the feasibility of deblending spectral line information in the IM regime. We consider a 3D IM survey, where multiple spectral lines at different redshifts are embedded in the same observing volume, and make use of the anisotropic shape of their respective power spectra when projected into a common comoving coordinate to disentangle the information. We consider deblending high-redshift [C II] signals from the brighter, lower-redshift $\mathrm{CO}$ interlopers. We use the halo-model and CIB measurement to construct expected $\mathrm{CO}$ and $[\mathrm{C} \mathrm{II}]$ templates across redshifts, and use the MCMC formalism to constrain power spectrum parameters. We show that this technique can reproduce the linear [C II] and $\mathrm{CO}$ power spectrum amplitudes, though with reduced signal-tonoise, given a range of $\mathrm{CO}$ signal strengths and noise levels. We establish an indicator to evaluate whether the fitted parameters are unbiased. Finally, we demonstrate the ability 


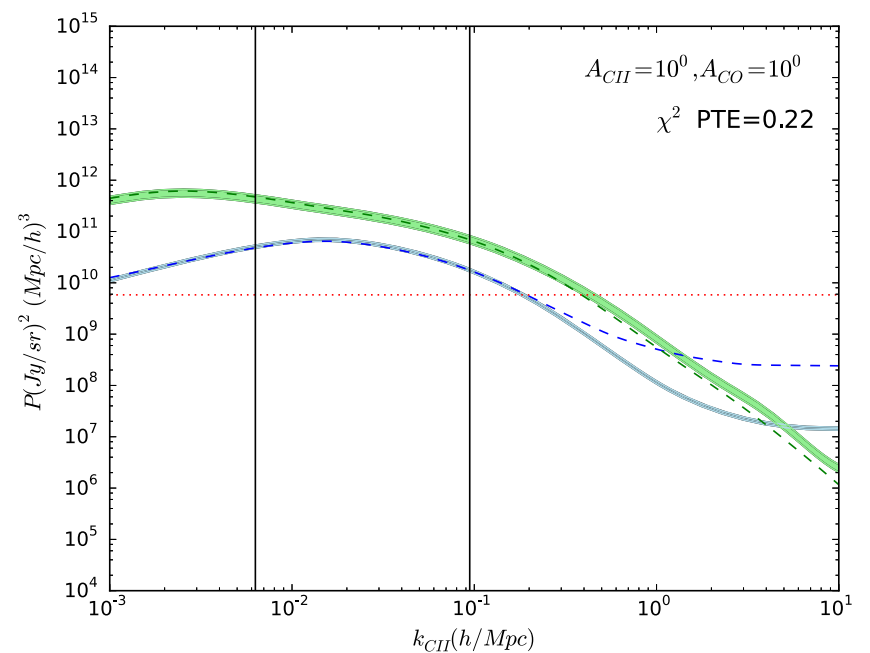

Figure 11. The ave-prj power spectrum of fiducial CIB model fitted with S15 template. The blue and green regions are the $68 \%$ confidence interval of [C II] and $\mathrm{CO}$ ave-prj power spectrum respectively. The dashed lines are the input [C II](blue) and $\mathrm{CO}$ (green) ave-prj power spectrum of the mock data. The red dotted line indicates $P_{\mathrm{sh}}^{\mathrm{C} I \mathrm{I}}+P_{\mathrm{sh}}^{\mathrm{CO}}+P_{n}$, which is the constant power spectrum in the data that contributes to the noise in $k$ space (see Equation (19)). The two vertical black lines are $k_{\mathrm{C} I I}^{\min }$ and $k_{\mathrm{CII}}^{\max }$ marking the $k$ space region we use in template fitting. The $\chi^{2}$ PTE for each MCMC fit is also provided.

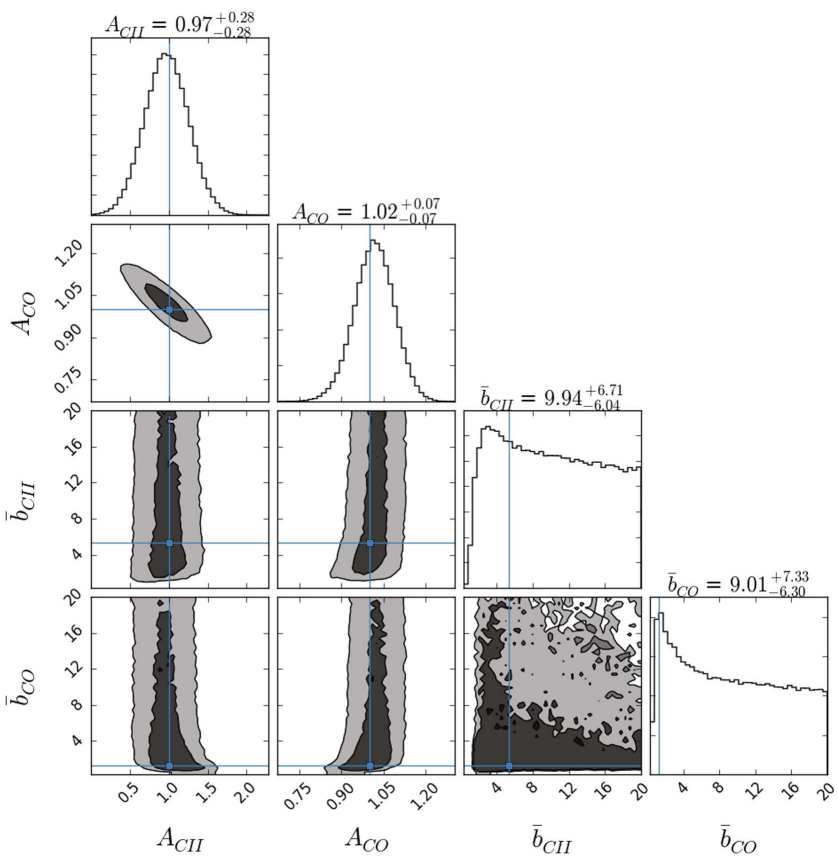

Figure 12. MCMC posterior distribution of the $\mathrm{S} 15$ case. The contours marked the $68 \%$ and $95 \%$ confidence interval in the parameter space. Crosshairs indicate the input value of the data: $\left\{A_{\mathrm{C} \text { II }}=1, A_{\mathrm{CO}}=1, \bar{b}_{\mathrm{C} \text { II }}=5.38, \bar{b}_{\mathrm{CO}}=1.29\right\}$.

of extracting [C II] in the presence of multiple, stronger $\mathrm{CO}$ foreground lines. The technique can be extended to other line blending problems to extract information of both signal and interlopers in an IM experiment.

We are grateful to the Time-Pilot collaboration for providing useful inputs throughout this work. We thank Phil Bull, Olivier Doré Tony Li, Adam Lidz, Roland de Putter, Paolo Serra, Chun-Hao To, and Heidi Hao-Yi Wu for helpful discussions and valuable comments on the manuscript. Y.-T.C. and T.-C.C.

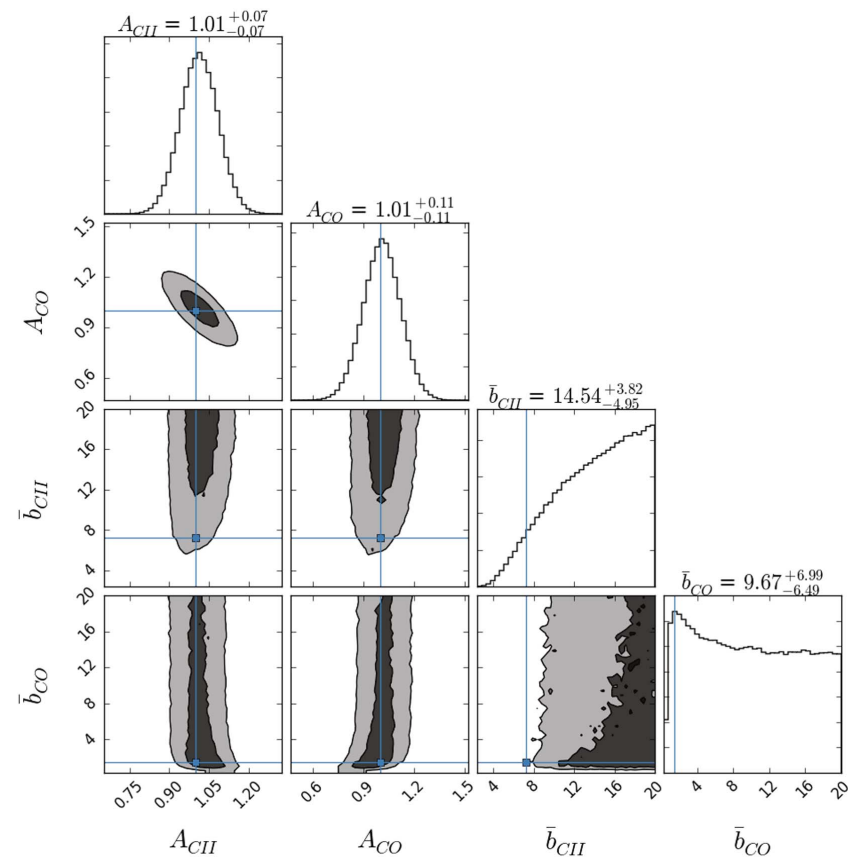

Figure 13. MCMC posterior distribution of the fiducial model with the lowest $k^{\|}$mode removed.

were supported in part by MoST grant 103-2112-M-001-002MY3. T.-C.C. gratefully acknowledges the hospitality of the Caltech OBSCOS group and the Jet Propulsion Laboratory, where part of this work was carried out.

\section{APPENDIX \\ POWER SPECTRUM MODELING}

\section{A.1. L $\mathrm{IR}_{\mathrm{R}}-M$ Relation: CIB Model}

In Planck Collaboration et al. (2014), the authors modeled the CIB emission as measured by Planck, and parametrized the CIB specific luminosity $L_{\nu}$ with the following three components.

1. A normalized spectral energy distribution, $\Theta(\nu, z)$, for all the galaxies:

$$
\begin{array}{ll}
\Theta(\nu, z) \propto \nu^{\beta} B_{\nu}\left(T_{d}(z)\right) ; & \nu<\nu_{0} \\
\Theta(\nu, z) \propto \nu^{-\gamma} ; & \nu \geqslant \nu_{0},
\end{array}
$$

where $B_{\nu}$ is the Planck function, and $T_{d}$ the redshiftdependent dust temperature:

$$
T_{d}=T_{0}(1+z)^{\alpha} .
$$

2. $L-M$ relation: they assumed the CIB luminosity is a $\log$ normal function $\Sigma$ of halo mass $M$ with peak mass $M_{\mathrm{eff}}$ and variance $\sigma_{L / M}^{2}$,

$$
\Sigma(M)=M \frac{1}{\left(2 \pi \sigma_{L / M}^{2}\right)^{1 / 2}} e^{-\left(\log _{10}(M)-\log _{10}\left(M_{\mathrm{eff}}\right)\right)^{2} / 2 \sigma_{L / M}^{2}} .
$$

3. Redshift evolution of the $L-M$ relation: the global normalization is parametrized by

$$
\Phi(z)=(1+z)^{\delta} .
$$

The $L-M$ ratio is assumed to increase with redshift (i.e., $\delta>0$ ). 


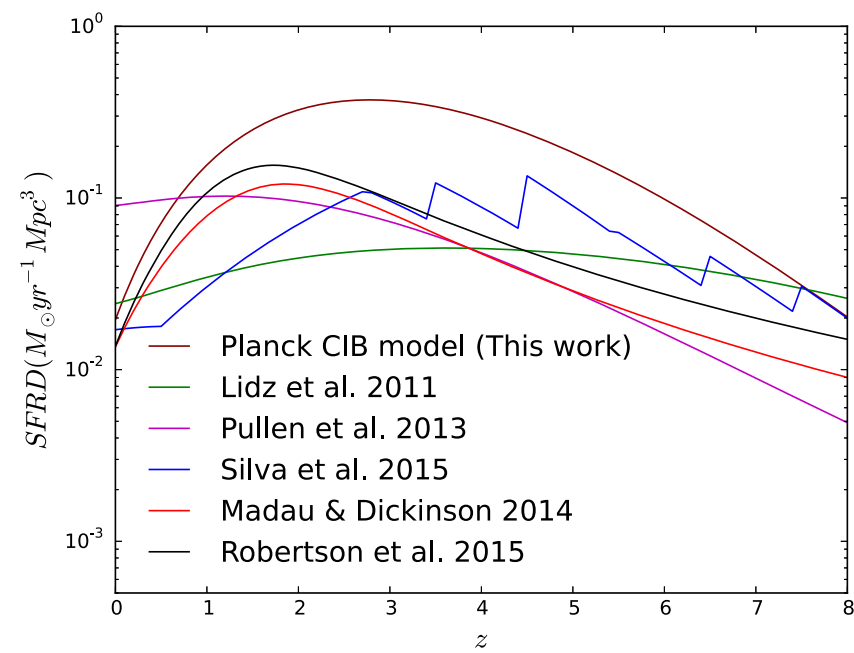

Figure 14. SFRD of the Planck CIB model (this work) comparing with the literature (Lidz et al. 2011; Pullen et al. 2013; Madau \& Dickinson 2014; Robertson et al. 2015; Silva et al. 2015).

Combining these three components, the $L_{\nu}-M$ relation can be written as

$$
L_{\nu}(M, z)=L_{0} \Phi(z) \Sigma(M) \Theta(\nu, z),
$$

with an overall normalization factor $L_{0}$.

With the Planck data, the authors constrained the model parameters as listed in Table 9 of Planck Collaboration et al. (2014). Here we adopt their best-fit values: $\{\alpha=0.36, \beta=$ $1.75, \gamma=1.7, \delta=3.6, \quad T_{0}=24.4 K, M_{\text {eff }}=$ $\left.10^{12.6} M_{\odot}, \sigma_{L / M}^{2}=0.5, \quad L_{0}=0.02 L_{\odot}\right\}$. We integrate Equation (27) over the wavelength range of $8-1000 \mu \mathrm{m}$ to obtain the total infrared luminosity $L_{\mathrm{IR}}$.

We can convert $L_{\mathrm{IR}}$ to SFR following Kennicutt (1998):

$$
S F R / L_{\mathrm{IR}}=1.7 \times 10^{-10} M_{\odot} \mathrm{yr}^{-1} L_{\odot}^{-1} .
$$

As a sanity check, we calculate the resulting star-formation rate density (SFRD) by integrating the SFR over halo mass,

$$
\operatorname{SFRD}(z)=\int_{M_{\text {min }}}^{M_{\max }} d M \frac{d N}{d M} \operatorname{SFR}(M, z),
$$

where $d N / d M$ is the halo mass function (Sheth \& Tormen 1999), and we take $M_{\min }=10^{8} M_{\odot} / \mathrm{h}, M_{\max }=10^{15} M_{\odot} /$ $h$. We use this integration range for all halo mass integration throughout this work.

For comparison, we calculate the SFRD from the SFR-M relation given by Lidz et al. (2011), Pullen et al. (2013), and Silva et al. (2015). We also consider the SFRD given by Madau \& Dickinson (2014) and Robertson et al. (2015), where the SFRD is modeled by the following four-parameter functional form

$$
\operatorname{SFRD}(z)=a \frac{(1+z)^{b}}{1+[(1+z) / c]^{d}}\left(M_{\odot} \mathrm{yr}^{-1} \mathrm{Mpc}^{-3}\right) .
$$

Madau \& Dickinson (2014) used the UV and IR galaxy counts and obtained the parameters $\{a, b, c, d\}=\{0.015,2.7,2.9$, $5.6\}$; while Robertson et al. (2015) used the joint constraint of galaxy counts and the CMB optical depth of $\tau=0.066 \pm 0.12$ from Planck Collaboration et al. (2015) and obtain $\{a, b, c$, $d\}=\{0.01376,3.26,2.59,5.68\}$.
The $\operatorname{SFRD}(z)$ of the aforementioned models are plotted in Figure 14. Lidz et al. (2011) and Pullen et al. (2013) use a simple scaling relation to model the SFR $-M$ relation, and do not reproduce the SFRD peak at $z \sim 2-3$. In Silva et al. (2015), the SFR- $M$ relation is fitted in several redshift bins with mock galaxy catalogs from De Lucia \& Blaizot (2007) and Guo et al. (2011). The discontinuous features in the SFRD curve are the boundaries of the redshift bins. They predict an SFRD peak at a higher redshift and the model does not agree well with galaxy counts at $z \sim 0-2$. The SFRD from the CIB model is broadly consistent in shape with that of galaxy counts, but the CIB model predicts a systematically higher amplitude, especially at high redshifts. This results in a higher [C II] power spectrum amplitude. Furthermore, the steep SFR $-M$ relation in the CIB model also results in a high bias factor for [C II]. We discuss the implications in Section 5.2.

\section{A.2. $L_{\mathrm{C} \text { II }}-S F R$ and $L_{\mathrm{CO}}-S F R$ Relation}

To connect $L_{\mathrm{C} \text { II }}$ to SFR, we adopt the following relation based on observations of nearby late-type galaxies (Boselli et al. 2002):

$$
L_{\mathrm{C} \text { II }}(M, z)=1.59 \times 10^{-3} L_{\mathrm{IR}},
$$

and use Equation (28) to convert $L_{\mathrm{IR}}$ to SFR.

For CO luminosity, we use the empirical relation from Wang et al. (2010):

$$
L_{\mathrm{CO}(1-0)}=3.2 \times 10^{4} L_{\odot}\left(\frac{\mathrm{SFR}}{M_{\odot} \mathrm{yr}^{-1}}\right)^{3 / 5} .
$$

For higher- $J$ rotational transitions, we assume a constant ratio between $L_{\mathrm{CO}(1-0)}$ and $L_{\mathrm{CO}(J-(J-1))}$, using Equation (16) of Obreschkow et al. (2009) and assuming an excitation temperature of $T_{e}=17 \mathrm{~K}$ for all the galaxies.

\section{REFERENCES}

Ali, Z. S., Parsons, A. R., Zheng, H., et al. 2015, ApJ, 809, 61 Boselli, A., Gavazzi, G., Lequeux, J., \& Pierini, D. 2002, A\&A, 385, 454 Bowman, J. D., Morales, M. F., \& Hewitt, J. N. 2009, ApJ, 695, 183 Breysse, P. C., Kovetz, E. D., \& Kamionkowski, M. 2014, MNRAS, 443, 3506 Breysse, P. C., Kovetz, E. D., \& Kamionkowski, M. 2015, MNRAS, 452, 3408 Carilli, C. L. 2011, ApJL, 730, L30

Chang, T. C., Gong, Y., Santos, M., et al. 2015, in Advancing Astrophysics with the Square Kilometre Array (AASKA14)

Chang, T.-C., Pen, U.-L., Bandura, K., \& Peterson, J. B. 2010, Natur, 466, 463

Chang, T.-C., Pen, U.-L., Peterson, J. B., \& McDonald, P. 2008, PhRvL, 100, 091303

Chapman, E., Abdalla, F. B., Harker, G., et al. 2012, MNRAS, 423, 2518

Comaschi, P., \& Ferrara, A. 2016, MNRAS, 455, 725

Cooray, A., \& Sheth, R. 2002, PhR, 372, 1

Crites, A. T., Bock, J. J., Bradford, C. M., et al. 2014, Proc. SPIE, 9153, $91531 \mathrm{~W}$

Croft, R. A. C., Miralda-Escudé, J., Zheng, Z., et al. 2016, MNRAS, 457, 3541 De Lucia, G., \& Blaizot, J. 2007, MNRAS, 375, 2

de Putter, R., Holder, G. P., Chang, T.-C., \& Dore, O. 2014, arXiv:1403.3727 Foreman-Mackey, D., Hogg, D. W., Lang, D., \& Goodman, J. 2013, PASP, 125,306

Furlanetto, S. R., Oh, S. P., \& Briggs, F. H. 2006, PhR, 433, 181

Gong, Y., Cooray, A., Silva, M., et al. 2012, ApJ, 745, 49

Gong, Y., Cooray, A., Silva, M. B., Santos, M. G., \& Lubin, P. 2011, ApJL, 728, L46

Gong, Y., Silva, M., Cooray, A., \& Santos, M. G. 2014, ApJ, 785, 72 Guo, Q., White, S., Boylan-Kolchin, M., et al. 2011, MNRAS, 413, 101 Kaiser, N. 1987, MNRAS, 227, 1

Keating, G. K., Bower, G. C., Marrone, D. P., et al. 2015, ApJ, 814, 140

Kennicutt, R. C., Jr. 1998, ApJ, 498, 541

Kogut, A., Dwek, E., \& Moseley, S. H. 2015, ApJ, 806, 234 
Li, T. Y., Wechsler, R. H., Devaraj, K., \& Church, S. E. 2016, ApJ, 817, 169 Lidz, A., Furlanetto, S. R., Oh, S. P., et al. 2011, ApJ, 741, 70

Lidz, A., \& Taylor, J. 2016, arXiv:1604.05737

Lidz, A., Zahn, O., Furlanetto, S. R., et al. 2009, ApJ, 690, 252

Liu, A., \& Tegmark, M. 2011, PhRvD, 83, 103006

Liu, A., \& Tegmark, M. 2012, MNRAS, 419, 3491

Madau, P., \& Dickinson, M. 2014, ARA\&A, 52, 415

Madau, P., Meiksin, A., \& Rees, M. J. 1997, ApJ, 475, 429

Mashian, N., Sternberg, A., \& Loeb, A. 2015, JCAP, 11, 028

Masui, K. W., Switzer, E. R., Banavar, N., et al. 2013, ApJL, 763, L20

Morales, M. F., Bowman, J. D., \& Hewitt, J. N. 2006, ApJ, 648, 767

Navarro, J. F., Frenk, C. S., \& White, S. D. M. 1996, ApJ, 462, 563

Obreschkow, D., Heywood, I., Klöckner, H.-R., \& Rawlings, S. 2009, ApJ, 702, 1321

Paciga, G., Albert, J. G., Bandura, K., et al. 2013, MNRAS, 433, 639

Parsons, A. R., Pober, J. C., Aguirre, J. E., et al. 2012, ApJ, 756, 165

Peacock, J. A. 1992, in New Insights into the Universe, Vol. 408 ed. V. J. Martinez, M. Portilla, \& D. Saez (Berlin: Springer), 1

Planck Collaboration, Ade, P. A. R., Aghanim, N., et al. 2014, A\&A, 571, A30

Planck Collaboration, Ade, P. A. R., Aghanim, N., et al. 2015, arXiv:1502. 01589

Pullen, A. R., Chang, T.-C., Doré, O., \& Lidz, A. 2013, ApJ, 768, 15
Pullen, A. R., Doré, O., \& Bock, J. 2014, ApJ, 786, 111

Righi, M., Hernández-Monteagudo, C., \& Sunyaev, R. A. 2008, A\&A, 489, 489

Robertson, B. E., Ellis, R. S., Furlanetto, S. R., \& Dunlop, J. S. 2015, ApJL, 802, L19

Schechter, P. 1976, ApJ, 203, 297

Scott, D., \& Rees, M. J. 1990, MNRAS, 247, 510

Sheth, R. K., \& Tormen, G. 1999, MNRAS, 308, 119

Silva, M., Santos, M. G., Cooray, A., \& Gong, Y. 2015, ApJ, 806, 209

Silva, M. B., Santos, M. G., Gong, Y., Cooray, A., \& Bock, J. 2013, ApJ, 763,132

Switzer, E. R., Chang, T.-C., Masui, K. W., Pen, U.-L., \& Voytek, T. C. 2015, ApJ, 815, 51

Switzer, E. R., Masui, K. W., Bandura, K., et al. 2013, MNRAS, 434, L46

Uzgil, B. D., Aguirre, J. E., Bradford, C. M., \& Lidz, A. 2014, ApJ, 793, 116

Visbal, E., \& Loeb, A. 2010, JCAP, 11, 016

Wang, R., Carilli, C. L., Neri, R., et al. 2010, ApJ, 714, 699

White, M. 2001, MNRAS, 321, 1

Wyithe, J. S. B., \& Loeb, A. 2008, MNRAS, 383, 606

Yue, B., Ferrara, A., Pallottini, A., Gallerani, S., \& Vallini, L. 2015, MNRAS, 450,3829 